\title{
Forman-Ricci Curvature for Hypergraphs
}

\author{
Wilmer Leal, ${ }^{1,2, *}$ Guillermo Restrepo, ${ }^{2,3}$ Peter F. Stadler, ${ }^{1,2,3,4,5,6}$ and Jürgen Jost ${ }^{2,6}$ \\ ${ }^{1}$ Bioinformatics Group, Department of Computer Science, \\ Universität Leipzig, Härtelstraße 16-18, 04107 Leipzig, Germany \\ ${ }^{2}$ Max Planck Institute for Mathematics in the Sciences, Inselstraße 22, 04103 Leipzig, Germany \\ ${ }^{3}$ Interdisciplinary Center for Bioinformatics, Universität Leipzig, Härtelstraße 16-18, 04107 Leipzig, Germany \\ ${ }^{4}$ Institute for Theoretical Chemistry, University of Vienna, Währingerstraße 17, 1090 Vienna, Austria \\ ${ }^{5}$ Facultad de Ciencias, Universidad Nacional de Colombia, KR 30-45 3, 111321, Bogotá, Colombia \\ ${ }^{6}$ The Santa Fe Institute, 1399 Hyde Park Rd., 87501, Santa Fe, New Mexico, USA
}

(Dated: November 20, 2018)

\begin{abstract}
In contrast to graph-based models for complex networks, hypergraphs are more general structures going beyond binary relations of graphs. For graphs, statistics gauging different aspects of their structures have been devised and there is undergoing research for devising them for hypergraphs. Forman-Ricci curvature is a statistics for graphs, which is based on Riemannian geometry, and that stresses the relational character of vertices in a network through the analysis of edges rather than vertices. In spite of the different applications of this curvature, it has not yet been formulated for hypergraphs. Here we devise the Forman-Ricci curvature for directed and undirected hypergraphs, where the curvature for graphs is a particular case. We report its upper and lower bounds and the respective bounds for the graph case. The curvature quantifies the trade-off between hyperedge(arc) size and the degree of participation of hyperedge(arc) vertices in other hyperedges(arcs). We calculated the curvature for two large networks: Wikipedia vote network and Escherichia coli metabolic network. In the first case the curvature is ruled by hyperedge size, while in the second by hyperedge degree. We found that the number of users involved in Wikipedia elections goes hand-in-hand with the participation of experienced users. The curvature values of the metabolic network allowed detecting redundant and bottle neck reactions. It is found that ADP phosphorilation is the metabolic bottle neck reaction but that the reverse reaction is not that central for the metabolism.
\end{abstract}

\section{INTRODUCTION}

Hypergraphs are used to model systems whose objects have not only binary relationships; instead, interactions simultaneously involve multiple members [1, 2]. Examples of these systems are found in physics, biology, chemistry, computer science, combinatorial optimization, scientometrics and several other fields [1,3-8]. Hypergraphs reduce to (ordinary) graphs when all relationships (hyperedges) are binary. Graphs have been widely used as a mathematical model for different systems and their mathematical properties have been extensively studied, which include devising statistics gauging aspects of their structures, such as vertex degree and its distributions, clustering coefficients, betweenness centrality and more recently Forman-Ricci curvature.

As hypergraphs are a generalization of graphs, several of the graph statistics have been extended to hypergraphs, e.g. vertex and hyperedge degrees, clustering coefficients [3, 9] and spectral properties [10]. Most of the commonly used quantities focus on vertices. As the crucial structure of a graph is, however, given by the set of its edges rather than by its vertices, we should systematically define and evaluate quantities assigned to the edges rather than to the vertices. In this paper we develop the Forman-Ricci curvature for hypergraphs (directed and undirected) and calculate it for networks of

*wilmer@bioinf.uni-leipzig.de different sizes and research fields.

\section{FORMAN-RICCI CURVATURE OF EDGES/ARCS IN GRAPHS}

Recently various notions of "curvature" have been proposed for graphs and other, more general, discrete structures and applied to detect various local or global properties of such structures [2, 11-19]. The name of "curvature" may seem somewhat strange in this context. In differential, and more abstractly, in Riemannian geometry, curvature has been found to encode and express local and global features of smooth manifolds equipped with metric tensors [20]. Those features themselves usually do not depend on an underlying smooth structure, and this has lead to abstract theories of generalized curvatures on metric spaces. On graphs, these generalized curvatures are particularly easy to define and to evaluate. They can also shed considerable light on other quantities that have been introduced in network analysis without such a clear conceptual background as those curvatures. The simplest among these generalized curvatures is the Ricci curvature introduced by Forman for simplicial complexes [21]. As graphs are one-dimensional simplicial complexes, we can readily evaluate this curvature. As explained in detail in Section II A, for an edge $e=\{i, j\}$ with vertices $i, j$ with degrees $d_{i}$ and $d_{j}$ (the degree of a vertex is the number of its neighbors, that is, of those other vertices that are directly connected to it by an edge), the Forman-Ricci curvature is simply $4-d_{i}-d_{j}$. The number 4 serves the 
purpose of normalization, to make the curvature of cycle graphs vanish. The minus signs are also conventional, to align this curvature with the Ricci curvature of Riemannian geometry. Thus, edges connecting vertices of large degree have very negative curvature values, and the first step in the analysis of an empirical network might consist in identifying the most negatively curved edges as the most important ones for the cohesion of the network or for the canalization and distribution of information or activity in the network.

Since the definition of the Forman-Ricci curvature of an edge in an undirected graph is so clear and simple, it can be readily generalized to, for instance, directed or weighted graphs, and also to structures in which more than two elements are related. Forman himself had introduced this curvature notion already for possibly weighted, simplicial complexes [21]. A simplicial complex is characterized by the requirement that whenever a collection of $k$ elements stands in relation, then this also holds for any subcollection. This leads to mathematically very nice properties, and simplicial complexes are basic structures in algebraic topology, but for the modelling and analysis of empirical data sets, we may want to relax or perhaps even completely abandon that condition. That leads us to hypergraphs, which are collections of vertices (undirected hypergraphs) or collections endowed with direction (directed hypergraphs). Examples of the former are elections, where a subset of voters is an election an the collection of elections constitutes the hypergraph. Chemical reactions $[3,22,23]$ and particle scatterings are instances of directed hypergraphs, where some starting materials are transformed into some products. For hypergraphs, in principle, various generalizations of the Forman-Ricci graph curvature are possible. It is a main contribution of this paper to identify that notion of Forman-Ricci curvature for (un)directed hypergraphs that is best adapted to their structure and to investigate its properties. We also apply this to concrete empirical hypernetworks, a social and a metabolic one.

In this section we briefly summarize the results of the Forman-Ricci curvature for graphs and then generalize the curvature for hypergraphs.

\section{A. Undirected graphs}

Let $G=(V, E)$ be a (multi)graph with vertex set $V$ and multiset of edges $E$. The Forman-Ricci curvature of an edge $e=\{i, j\} \in E$, as introduced in [11], is given by:

$$
F(e)=w_{e}\left(\frac{w_{i}}{w_{e}}+\frac{w_{j}}{w_{e}}-\sum_{e_{l} \sim i} \frac{w_{i}}{\sqrt{w_{e} w_{e_{l}}}}-\sum_{e_{l} \sim j} \frac{w_{j}}{\sqrt{w_{e} w_{e_{l}}}}\right)
$$

where $w_{e}$ denotes the weight of the edge $e, w_{i}$ and $w_{j}$ are the weights of vertices $i$ and $j$, respectively. The sums over $e_{l} \sim k$ run over all edges $e_{l}$ incident on the vertex $k$ excluding $e$. The curvature for the unweighted multigraph, with vertex and edge weights set to 1 , is given by [2]

$$
F(e)=4-d_{i}-d_{j}
$$

where $d_{k}$ is the vertex degree of $k$. Defining $D=\sum_{k \in e} d_{k}$ we have

$$
F(e)=4-D
$$

As a multigraph may have repeated edges, whose number is independent of the number of vertices, the bounds for $F(e)$ shall be expressed as a function of the known number of edges, namely, $|E|$. Therefore, $2(2-|E|) \leq$ $F(e) \leq 2$. The lower bound is attained when $d_{k}=|E|$ for every $k \in e$, therefore $D=2|E|$ (Figure 1a). In turn $F(e)=2$, for an isolated edge $e$ (Figure 1c). In contrast to the multigraph case, for simple unweighted graphs, the lower bound can be expressed as a function of the number of vertices: $2(3-|V|) \leq F(e)$, which is obtained for $d_{k}=|V|-1$ for every $k \in e$, i.e., $D=2(|V|-1)$ (Figure $1 b)$. As for multigraphs, $F(e)$ reaches its maximum value $(F(e)=2)$ for an isolated edge (Figure 1c).

As seen in Figure 1, Forman-Ricci curvature quantifies the degree of spread of the vertices in $e$, from maximum spread (corresponding to $\min F(e)$ ) to minimum spread (attained when $\max F(e)$ ).

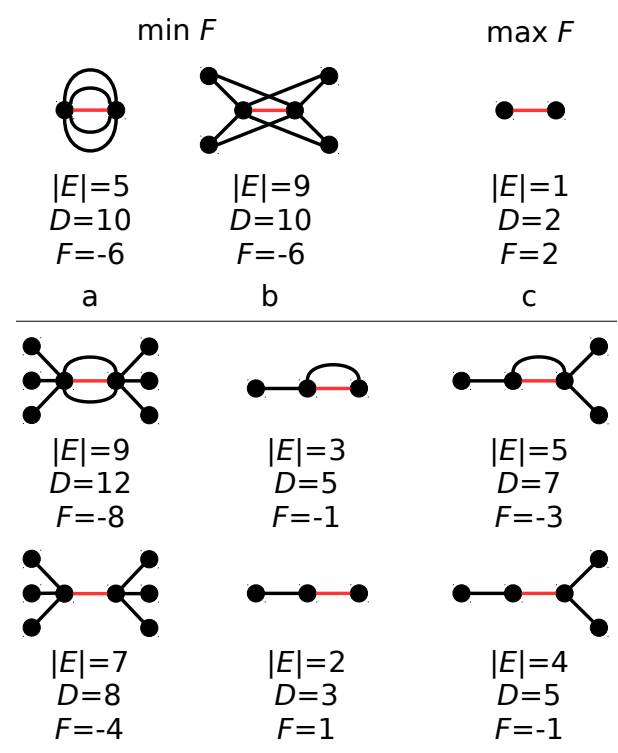

FIG. 1. Forman-Ricci curvatures $F(e)$ calculated for the red edge $e$ of the depicted undirected graphs.

\section{B. Directed graphs}

Here we are interested in an unweighted directed multigraph $G=(V, E)$, where $e=(i, j) \in E$ is an arc (directed edge), and $i, j \in V$. Equation 2 indicates that the curvature of an edge depends on the degree of its vertices. As in a simple directed graph the degree can be split into 
in- and out-degree. The curvature of $e=(i, j)$ is defined in terms of in- and out-degrees as well [17]. There are different possibilities for the realization of the curvature, depending on the meaning one assigns to it. Here we emphasize the directed spread or flow through e, i.e., following the direction of the arc. Therefore, we consider the incoming arcs on $i$ (in-degree of $i, \operatorname{in}(i)$ ) and the outgoing arcs from $j$ (out-degree of $j$, out $(j)$ ). When we separate the curvature in (2) into the contribution $2-d_{i}$ of $i$ and $2-d_{j}$ of $j$ and also note that the edge $e$ counts for the degrees of $i$ and $j$, but neither for the in-degree of $i$ nor for the out-degree of $j$, then a curvature accounting for the in-flow at $i(F(\rightarrow e))$ and another for the out-flow at $j\left(F\left(e_{\rightarrow}\right)\right)$ is defined as

$$
\begin{aligned}
& F(\rightarrow e)=1-\operatorname{in}(i) \\
& F\left(e_{\rightarrow}\right)=1-\operatorname{out}(j) .
\end{aligned}
$$

Both are bounded below by $2-|E|$ for in $(i)=\operatorname{out}(j)=$ $|E|-1$, and bounded above by 1 when $\operatorname{in}(i)=\operatorname{out}(j)=0$ (Figure 2a). For the simple directed graph the lower bound for both, in- and out-flow, is $2-|V|$, for in $(i)=$ $\operatorname{out}(j)=|V|-1$ (Figure $2 \mathrm{~b})$. The upper bound is reached, in both cases, when $\operatorname{in}(i)=\operatorname{out}(j)=0$ (Figure $2 \mathrm{c})$. The curvature accounting for the flow through $e=(i, j)$ is then given by

$$
\begin{aligned}
F\left(\rightarrow e_{\rightarrow}\right) & =F(\rightarrow e)+F\left(e_{\rightarrow}\right) \\
& =2-\operatorname{in}(i)-\operatorname{out}(j)
\end{aligned}
$$

where $2(2-|E|) \leq F\left(\rightarrow e_{\rightarrow}\right) \leq 2$ for the multigraph case and $2(2-|V|) \leq F\left(\rightarrow e_{\rightarrow}\right) \leq 2$ in the simple graph case. Figure 2c shows the case where $F\left(\rightarrow e_{\rightarrow}\right)=2$. Some further examples of calculations of curvatures $F\left(\rightarrow e_{\rightarrow}\right)$ are shown in Figure 2.

If the flow-loss along $e$ is to be considered, two additional curvatures are calculated that account for the flow loss at $i(F(\leftarrow e))$ and at $j\left(F\left(e_{\leftarrow}\right)\right)$. Thus

$$
\begin{aligned}
& F(\leftarrow e)=1-\operatorname{out}(i) \\
& F\left(e_{\leftarrow}\right)=1-\operatorname{in}(j)
\end{aligned}
$$

both bounded below by $1-|E|$, for $\operatorname{out}(i)=\operatorname{in}(j)=$ $|E|$, and bounded above by 0 for $\operatorname{out}(i)=\operatorname{in}(j)=1$ (Figure 2d). For the simple directed graph we have $2-$ $|V| \leq F(\leftarrow e) \leq 0$ and $2-|V| \leq F\left(e_{\leftarrow}\right) \leq 0$. Hence, the curvature for the flow-loss along $e=(i, j)$ is

$$
\begin{aligned}
F\left(\leftarrow e_{\leftarrow}\right) & =F(\leftarrow e)+F\left(e_{\leftarrow}\right) \\
& =2-\operatorname{out}(i)-\operatorname{in}(j)
\end{aligned}
$$

where $2(1-|E|) \leq F\left(\leftarrow e_{\leftarrow}\right) \leq 0$ (Figures 2a-e) holds in the multigraph case and $2(2-|V|) \leq F\left(\rightarrow e_{\rightarrow}\right) \leq 0$ in the simple graph case. Some further examples are shown in Figure 2.

A curvature accounting for the total flow over $e$ is then computed as

$$
F(e)=F\left(\rightarrow e_{\rightarrow}\right)+F\left(\leftarrow e_{\leftarrow}\right)
$$

In the following section we extend the Forman-Ricci curvature to hypergraphs.

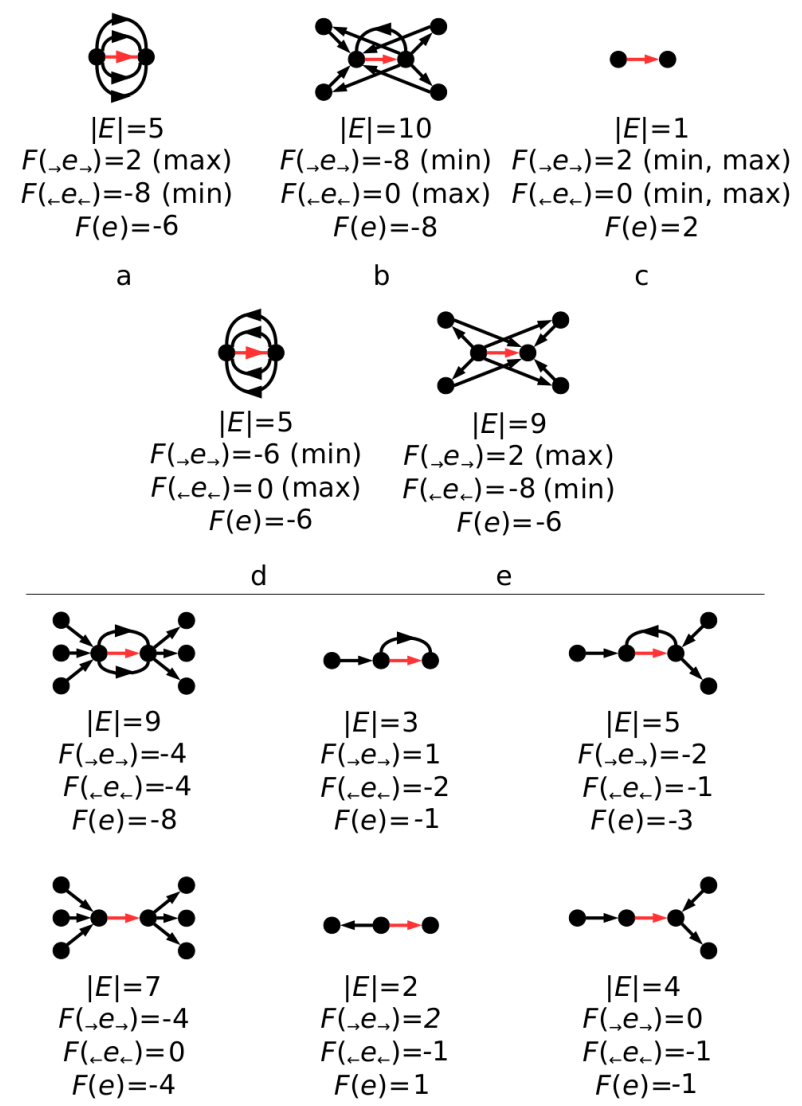

FIG. 2. Forman-Ricci curvatures $F\left(\rightarrow e_{\rightarrow}\right), F\left(\leftarrow e_{\leftarrow}\right)$, and $F(e)$ calculated for the red arc $e$ of the depicted directed graphs.

\section{FORMAN-RICCI CURVATURE OF HYPER(EDGES/ARCS) IN HYPERGRAPHS}

Given a set of vertices $V$, a graph is a collection of subsets (edges) of $V$, all of which comprise only two elements. If we call the cardinality of each subset its size, then a graph is a collection of subsets of size two. In a hypergraph, the size of the subsets is no longer restricted, and subsets of any size are allowed.

\section{A. Undirected hypergraphs}

An undirected hypergraph $H=(V, E)$ consists of a set $V$ of vertices and a multiset $E$ of subsets of $V$, called hyperedges, such that $e \subseteq V$, i.e. $|e| \leq|V|$, for $e \in E$. Some examples of hypergraphs are shown in Figure 3.

Separating the contributions of vertices $i$ and $j$ in Equation 1, it can be rewritten as:

$$
F(e)=w_{e}\left[\left(\frac{w_{i}}{w_{e}}-\sum_{e_{l} \sim i} \frac{w_{i}}{\sqrt{w_{e} w_{e_{l}}}}\right)+\left(\frac{w_{j}}{w_{e}}-\sum_{e_{l} \sim j} \frac{w_{j}}{\sqrt{w_{e} w_{e_{l}}}}\right)\right]
$$


furthermore,

$$
F(e)=w_{e}\left[\sum_{k \in e}\left(\frac{w_{k}}{w_{e}}-\sum_{e_{l} \sim k} \frac{w_{k}}{\sqrt{w_{e} w_{e_{l}}}}\right)\right]
$$

Since Equation 10 no longer restricts $e$ to size two, we present it as the Forman-Ricci curvature of the hyperedge $e$. For the unweighted hypergraph, where all vertex weights are equal to 1 , this expression simplifies to

$$
F(e)=\sum_{k \in e}\left(2-d_{k}\right)=2|e|-\sum_{k \in e} d_{k}=2|e|-D
$$

which is bounded below by $|e|(2-|E|)$ when $d_{k}=|E|$ for every $k \in e$, and bounded above by 1 when $D=|e|$. In other words, the minimum curvature occurs when every vertex in $e$ belongs to each hyperedge (Figures 3a,b); the maximum is attained for an isolated hyperedge (Figure 3c).

For the particular case of simple hypergraphs, we therefore have the lower bound $2|e|\left(1-2^{|V|-2}\right)$ when $d_{k}=|\mathcal{P}(V \backslash\{k\})|$ for every $k \in e$, and the upper bound $|V|$, when $E=\{V\}$. Note that in hypergraphs $|e| \leq|V|$, therefore the minimum value $|e|$ may reach 1 , unlike graphs. In such a case, $2\left(1-2^{|V|-2}\right) \leq F(e) \leq|V|$. Some further examples of curvature for hypergraphs are shown in Figure 3.

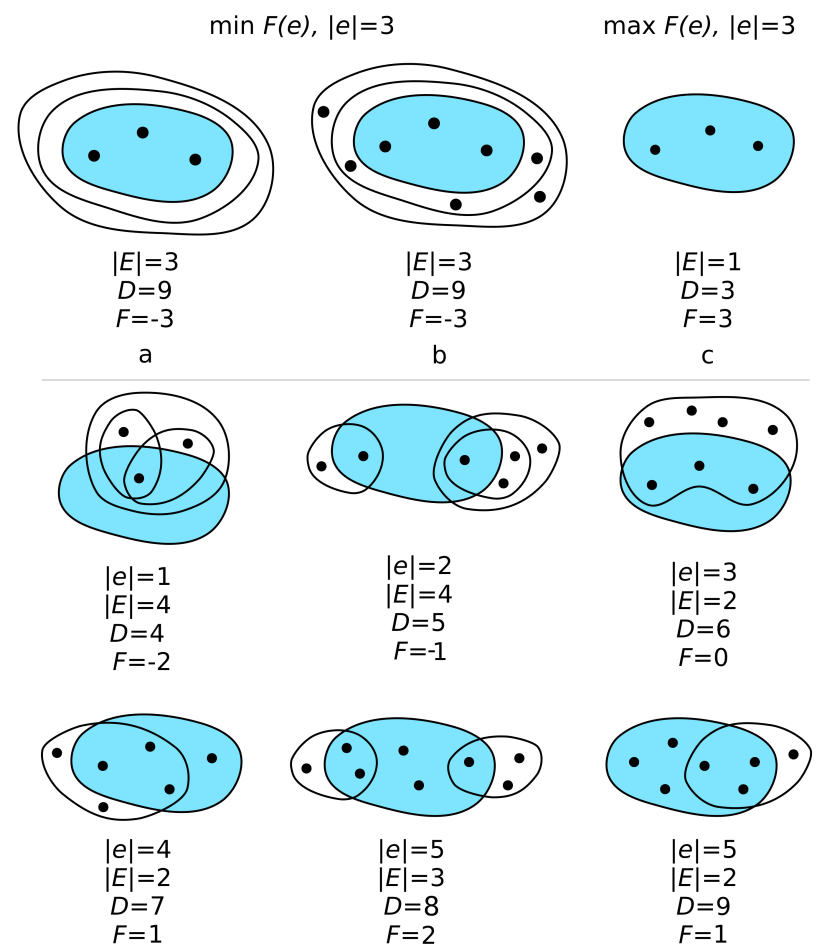

FIG. 3. Forman-Ricci curvatures $F(e)$ calculated for the blue hyperedge $e$ of the depicted hypergraphs.

\section{B. Directed hypergraphs}

In a directed hypergraph, each hyperedge is composed of two subsets of vertices: the tail and the head of the hyperedge. Formally, we say that a directed hypergraph $H$ is the couple $(V, E)$ with $V$ a set of vertices and $E$ a multiset of hyperarcs. A hyperarc is a pair $e=\left(e_{i}, e_{j}\right)$, where $e_{i} \subseteq V$ and $e_{j} \subseteq V$ are called its tail and its head, respectively. Figure 4 depicts some examples of diercted hypergraphs, where the sets $e_{i}$ and $e_{j}$ are highlighted.

Starting from the definitions of curvature for an arc in the directed graph case (Equation 4), we introduce the curvatures $F(\rightarrow e)$ and $F\left(e_{\rightarrow}\right)$ for a hyperarc as

$$
\begin{aligned}
& F(\rightarrow e)=\left|e_{i}\right|-\sum_{i \in e_{i}} \operatorname{in}(i) \\
& F\left(e_{\rightarrow}\right)=\left|e_{j}\right|-\sum_{j \in e_{j}} \operatorname{out}(j)
\end{aligned}
$$

with bounds $\left|e_{i}\right|(1-|E|) \leq F(\rightarrow e) \leq\left|e_{i}\right|$ and $\left|e_{j}\right|(1-$ $|E|) \leq F\left(e_{\rightarrow}\right) \leq\left|e_{j}\right|$. For the simple directed hypergraphs, we have $\left|e_{i}\right|\left(1-2^{|V|-1}\right) \leq F(\rightarrow e) \leq\left|e_{i}\right|$ and $\left|e_{j}\right|\left(1-2^{|V|-1}\right) \leq F\left(e_{\rightarrow}\right) \leq\left|e_{j}\right|$. With $F(\rightarrow e)$ and $F\left(e_{\rightarrow}\right)$ at hand, we define the curvature for the flow through $e=\left(e_{i}, e_{j}\right)$ as:

$$
\begin{aligned}
F\left(\rightarrow e_{\rightarrow}\right) & =F(\rightarrow e)+F\left(e_{\rightarrow}\right) \\
& =\left|e_{i}\right|+\left|e_{j}\right|-\sum_{i \in e_{i}} \operatorname{in}(i)-\sum_{j \in e_{j}} \operatorname{out}(j)
\end{aligned}
$$

with bounds $(1-|E|)\left(\left|e_{i}\right|+\left|e_{j}\right|\right) \leq F\left(\rightarrow e_{\rightarrow}\right) \leq\left|e_{i}\right|+\left|e_{j}\right|$ in the general case and $\left(1-2^{|V|}\right)\left(\left|e_{i}\right|+\left|e_{j}\right|\right) \leq F\left(\rightarrow e_{\rightarrow}\right) \leq$ $\left|e_{i}\right|+\left|e_{j}\right|$ for the simple directed hypergraph (Figure 4$)$. Note that if $|e|$ is allowed to have its minimum value of 1 , then $\left|e_{k}\right|=1$ and $2(1-|E|) \leq F\left(\rightarrow e_{\rightarrow}\right) \leq 2$. Some examples of curvature values for directed hypergraphs are shown in Figure 4.

The respective flow-loss curvatures are:

$$
\begin{aligned}
& F(\leftarrow e)=\left|e_{i}\right|-\sum_{i \in e_{i}} \operatorname{out}(i) \\
& F\left(e_{\leftarrow}\right)=\left|e_{j}\right|-\sum_{j \in e_{j}} \operatorname{in}(j)
\end{aligned}
$$

with bounds $\left|e_{i}\right|(1-|E|) \leq F(\leftarrow e) \leq 0$ and $\left|e_{j}\right|(1-$ $|E|) \leq F\left(e_{\leftarrow}\right) \leq 0$ in the general case and $\left|e_{i}\right|\left(1-2^{|V|}\right) \leq$ $F(\leftarrow e) \leq 0$ and $\left|e_{j}\right|\left(1-2^{|V|}\right) \leq F\left(e_{\leftarrow}\right) \leq 0$ for the simple directed hypergraphs.

Equation 14 yields the flow-loss curvature

$$
\begin{aligned}
F\left(\leftarrow e_{\leftarrow}\right) & =F(\leftarrow e)+F\left(e_{\leftarrow}\right) \\
& =\left|e_{i}\right|+\left|e_{j}\right|-\sum_{i \in e_{i}} \operatorname{out}(i)-\sum_{j \in e_{j}} \operatorname{in}(j)
\end{aligned}
$$

with bounds $(1-|E|)\left(\left|e_{i}\right|+\left|e_{j}\right|\right) \leq F\left(\leftarrow e_{\leftarrow}\right) \leq 0$, which becomes $\left(1-2^{|V|-1}\right)\left(\left|e_{i}\right|+\left|e_{j}\right|\right) \leq F\left(\leftarrow e_{\leftarrow}\right) \leq 0$ for simple directed hypergraphs. 


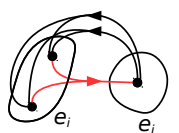

$|E|=3$

$F(\rightarrow e)=-2$

$F\left(e_{\rightarrow}\right)=-1$

$F(๘ e)=0(\max )$

$F\left(e_{\leftarrow}\right)=0(\max )$

$F\left(e_{\rightarrow}\right)=-3$

$F\left(\leftarrow e_{\leftarrow}\right)=0$

$F(e)=0$

a

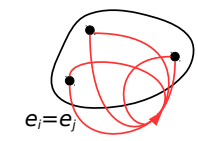

$|E|=1$

$F(\rightarrow e)=0(\min )$ $F\left(e_{\rightarrow}\right)=0(\mathrm{~min})$

$F\left(\_e\right)=0$ (min, max)

$F\left(e_{\leftarrow}\right)=0(\min , \max )$ $F\left({ }_{\rightarrow} e_{\rightarrow}\right)=0(\min )$

$F\left({ }_{\leftarrow} e_{\leftarrow}\right)=0(\min , \max )$

$F(e)=0$

b

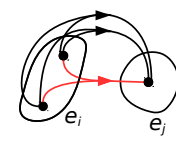

$|E|=3$

$F(\rightarrow e)=2(\max )$

$F\left(e_{\rightarrow}\right)=1(\max )$

$F\left(\_e\right)=-1$

$F\left(e_{+}\right)=-2(\min )$

$F\left({ }_{\rightarrow} e_{\rightarrow}\right)=3(\max )$

$F\left(e_{+} e_{+}\right)=-3$

$F(e)=0$

d

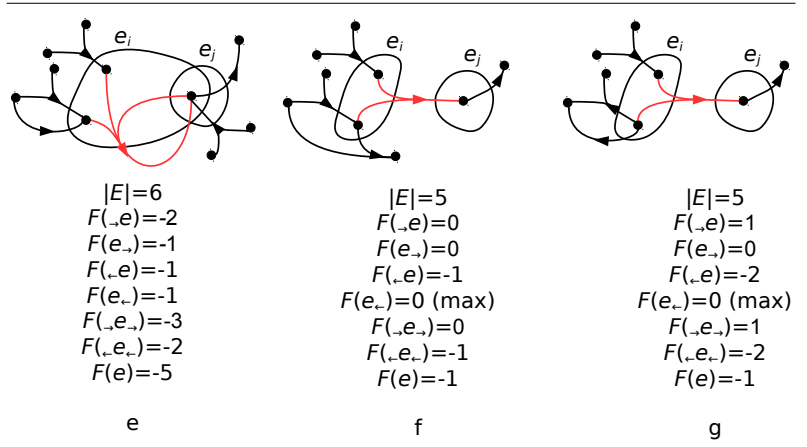

FIG. 4. Forman-Ricci curvatures $F\left(\rightarrow e_{\rightarrow}\right), F\left(\leftarrow e_{\leftarrow}\right)$, and $F(e)$ calculated for the red hyperarc $e$, connecting vertices in $e_{i}$ with those in $e_{j}$, of the depicted hypergraphs.

In the following section we calculate the Forman-Ricci curvature for different cases that can be modelled as hypergraphs. Several applications of the Forman-Ricci curvature for the graph case are found in references $[2,11-$ $19]$.

\section{APPLICATIONS TO EMPIRICAL NETWORKS}

\section{Wikipedia Voting Network}

Wikipedia is an encyclopedia written by volunteers. A small part of these users are administrators, who besides being active, regular long-term Wikipedia contributors, have gained the general trust of the community and have taken on technical maintenance duties. A user becomes an administrator when a request for adminship is issued and the Wikipedia community via a public vote decides who to promote to administrator. Users can either submit their own requests for adminship or may be nomi- nated by other users. Using the January 32008 dump of Wikipedia page edit history [24], Leskovec et al. [25] extracted 2,794 elections (hyperedges in our setting) and 7,066 users (vertices) participating in the elections (either casting a vote or being voted on). We calculated the curvature for the resulting undirected hypergraph.

Figure 5 shows the distribution of hyperedge size and of vertex degree. The data show that many of the elections involve a single user, although elections with 2-20 users are also common. There are few elections with more than 100 users, the largest one including 370 users (Figure 5a). The participation in elections is heavy-tailed distributed (Figure 5b), with most of the users participating in a single election and very few taking part in about a thousand elections. The curvature values are mostly negative (Figure 5c), indicating (i) the absence of elections with unexperienced users $(\max F(e) \neq|e|)$, i.e., all elections at least include a user that takes part in at least one other election; and (ii) for most elections the number of elections in which users take part is greater than their number of voting users $(D>|e|$ in Equation 3). The minimum curvature value $(-3,112)$ is far from the lower bound $(-19,728,272$, calculated with $|e|=7,066)$. This reflects the fact that most users are experts in limited fields only.

To have some insight about the effects of hyperedge sizes and number of incident hyperedges on curvature, we analyzed their distributions over the span of curvature values (Figures 5d-f). Figure 5d shows that the more spread the election, i.e. involving users that vote in other elections, the larger the number of users voting. Figures 5e and $\mathrm{f}$ show that, in average, elections overlap with a low number of other elections (low number of incident elections). Thus, the curvature values are mainly ruled by hyperedge size rather than by incident hyperedges.

\section{Metabolic Network of Escherichia coli}

The metabolism of Escherichia coli is one of the most studied and best characterized among bacteria. Here we model the metabolism K-12 (iJR904 GSM/GPR) [26] of this bacterium as a directed hypergraph whose vertices are the metabolites (chemical species). Each chemical reaction is represented as a hyperarc $e$, whose educts (starting materials) correspond to $e_{i}$ and products to $e_{j}$. There are $|V|=625$ metabolites and $|E|=1,176$ reactions accounting for 686 non-reversible and 245 reversible ones. These latter reactions, denoted by $e_{i} \leftrightarrow e_{j}$ have been included as "forward" $\left(e_{i} \rightarrow e_{j}\right)$ and "backward" $\left(e_{j} \rightarrow e_{i}\right)$ reactions. All curvatures (Equations 12 to 15$)$ and related calculations are gathered in the Supplementary Material.

As expected for chemical reactions, typically there are not more than three educts and three products (Figure $6 a)$. The curvature values therefore vary little in response to hyperarc size, but rather depend more on the degree of vertices in $e_{i}$ and $e_{j}$. Note that these degrees result, 

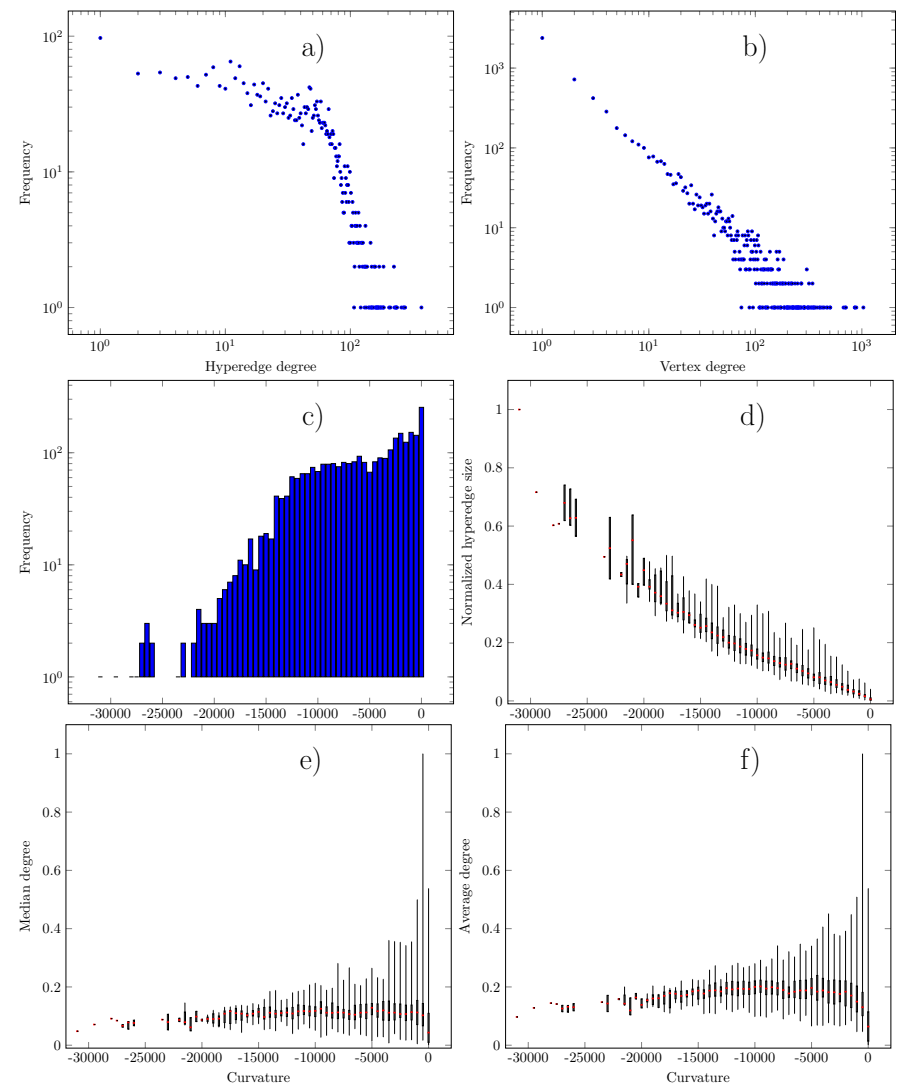

FIG. 5. Voting Wikipedia: Distribution of a) hyperedge size (size of elections) and b) vertex degree (participation of users in elections). c) Histogram of curvature values with bins of 10 units. Box-plots of d) normalized hyperedge sizes, e) median, and f) average hyperedge degrees corresponding to each curvature bin of $\mathrm{c}$ ).

respectively, from the summation over vertex degrees of educts and of products (Equations 12 to 15). The distribution and educts and products degrees is shown in Figure 6. The participation of educts and products in reactions does not yield a smooth distribution, as indicated by the gaps present in Figure $6 \mathrm{~b}, \mathrm{c}$. The production of educts (Figure 6b) shows a large group of reactions whose educts are synthesized by less than 200 reactions and another group where they are obtained by more than 450 reactions. Likewise, there are two groups of reactions with different levels of use of their products (Figure 6c); one group has reactions whose products are used in less than 100 reactions and another with more than 300 reactions taking their products as starting materials.

The synthesis of products and the use of educts (Figures $6 \mathrm{~d}$ and e), shows also a discontinuous participation of substrates in reactions. There are two groups of reactions according to the number of reactions synthesizing their products: one with reactions whose products are obtained by less than 200 reactions and another by more than 450 reactions (Figure 6d). Likewise, there are various groups of reactions according to the use of their educts, from some which are seldom used to some others with about 170, 230, and more than 330 uses (Figure $6 e)$.
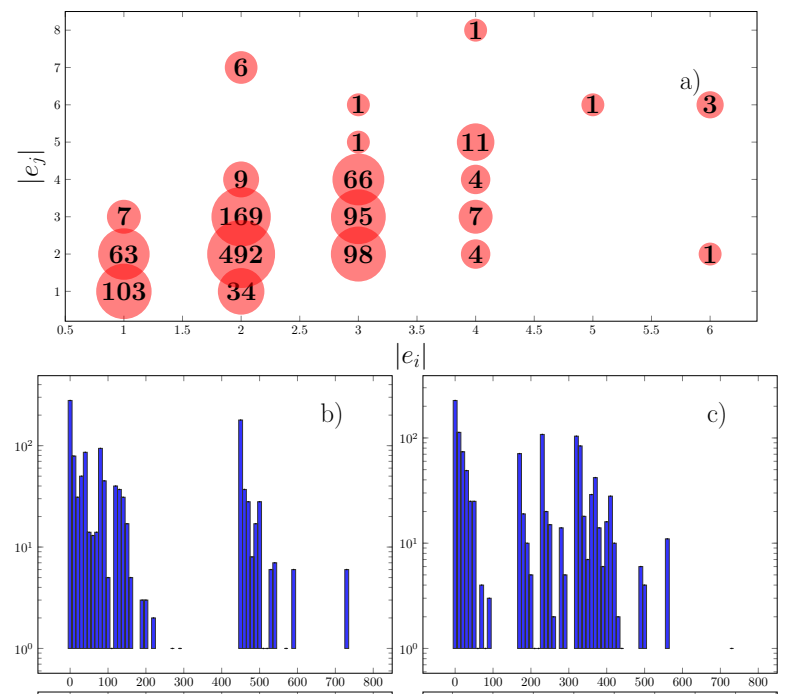

$\left|e_{i}\right|$
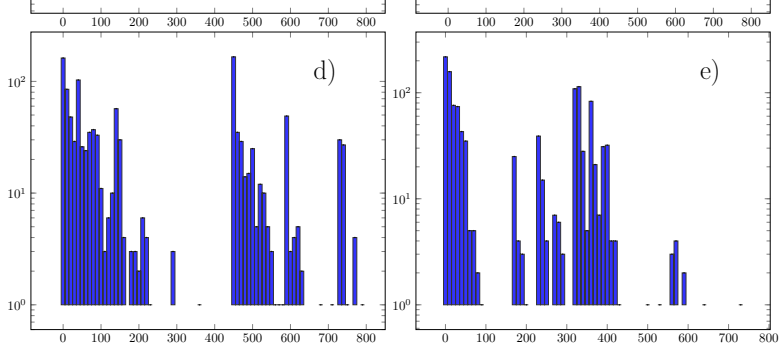

FIG. 6. Metabolic network: a) Scatter plot of sizes of educts $\left(\left|e_{i}\right|\right)$ and products $\left(\left|e_{j}\right|\right)$, where circle radii correspond to $\log f / \log 100$, being $f$ the frequency of appearance of the couple $\left(\left|e_{i}\right|,\left|e_{j}\right|\right)$ in the reactions. Numbers inside circles correspond to $f$. Distribution of b) $\sum_{i \in e_{i}} \operatorname{in}(i)$, c) $\sum_{i \in e_{i}} \operatorname{out}(i)$, d) $\sum_{j \in e_{j}} \operatorname{in}(j)$, and $\sum_{j \in e_{j}} \operatorname{out}(j)$.

The extent to which the educts of the reaction $e$ are produced from other reactions is measured by $F(\rightarrow e)$. The more reactions lead to the educts of $e$, the more negative $F(\rightarrow e)$ becomes (Figure 7a). The theoretical bounds of $F(\rightarrow e)$, assuming $\max \left|e_{i}\right|=625$ are $-734,375 \leq F(\rightarrow e) \leq 625$. However, more realistic bounds are $-7,050 \leq F(\rightarrow e) \leq 6$, which results from taking the actual max $\left|e_{i}\right|=6$ (Figure $6 \mathrm{a}$ ). We found that $\min F(\rightarrow e)=-735$, which is attained by four reactions, with four educts (all substrate abbreviations are included in the Appendix (Table I)):

$\mathrm{adp}+\mathrm{h}+\mathrm{malcoa}+\mathrm{pi} \rightarrow \mathrm{accoa}+\mathrm{atp}+\mathrm{hco} 3$

$\mathrm{adp}+\mathrm{h}+\mathrm{pi}+25 \mathrm{aics} \rightarrow \mathrm{asp} \_$L + atp $+5 \mathrm{aizc}$

$\mathrm{adp}+\mathrm{dtbt}+\mathrm{h}+\mathrm{pi} \rightarrow \mathrm{atp}+\mathrm{co} 2+$ dann

atp + gar $+\mathrm{h}+\mathrm{pi} \rightarrow \mathrm{atp}+\mathrm{gly}+$ pram

These reactions are those whose educts are the most synthesized of all the metabolic reactions of E. coli $(63 \%$ of the reactions produce their educts). In three of them atp is synthesized from adp, which shows the well-known 
central metabolic role of atp [27, 28].

$\max F(\rightarrow e)=1$ corresponds to a single reaction: cyan+tsul $\rightarrow \mathrm{h}+$ so3+tcynt, where only one of its two educts is a product of a single reaction: atp + h2o + tsul $\rightarrow$ adp $+\mathrm{h}+\mathrm{pi}+$ tsul.

Figure 7 a shows that the most frequent curvature value is 0 (for 73 reactions), i.e. $6 \%$ of the reactions have a trade-off between the number of educts and the number of reactions producing them; most of the remaining reactions have more ways to produce their educts than the number of educts. It is also found that there are almost no reactions with curvatures between -200 and -450 , indicating that educts of reactions are mainly obtained either by less than 200 reactions (less than $17 \%$ of the reactions) or by 450 to 600 reactions (38 to $51 \%$ of the reactions). This is a consequence of the heavy-tailed in-degree distribution of substrates [27].

Figure $7 \mathrm{~b}$ shows the curvature values $F\left(e_{\rightarrow}\right)$, which quantify the extent to which products of reactions are used in further reactions as educts. By taking $\max \left|e_{j}\right|=$ 8 (Figure 6a) this curvature takes values $-9,400 \leq$ $F\left(e_{\rightarrow}\right) \leq 8$. The actual $\min F\left(e_{\rightarrow}\right)=-729$, for $\mathrm{adp}+\mathrm{h}+\mathrm{pi} \rightarrow \mathrm{atp}+\mathrm{h}+\mathrm{h} 2$ o, i.e., this is the reaction whose three products are most used in other reactions as starting materials (used in $62 \%$ of the reactions). In contrast, there are four reactions with $\max F\left(e_{\rightarrow}\right)=1$ :

agpe_EC + pg_EC $\rightarrow$ apg_EC+g3pe

agpc_EC + pg_EC $\rightarrow$ apg_EC+g3pc

agpg_EC + pg_EC $\rightarrow$ apg_EC + g3pg

udpgal $\rightarrow$ udpgalfur

Hence, for those three reactions with two products, these substrates are only used in a further reaction as educts, while udpgalfur is not further used, i.e. it is a metabolic "dead-end" [26]. As most of the reactions (96\%) have negative values of $F\left(e_{\rightarrow}\right)$, this indicates the efficient use of reaction products [28], which can be divided into two regimes. For about half of the reactions their products are used in no more than $9 \%$ of the reactions and about $40 \%$ of the reactions have products that are used in more than a quarter of the reactions. This is a consequence of the heavy-tailed distribution, this time, of the outdegrees of the substrates [27].

$F(\rightarrow e)$ showed that for most of the reactions their educts are produced by other reactions and $F\left(e_{\rightarrow}\right)$ that the products are used in several other reactions. The question that arises whether those popular educts are connected through reactions with the popular products is positively answered by $F\left(\rightarrow e_{\rightarrow}\right)$, which takes negative values for most of the reactions. The $\min F\left(\rightarrow e_{\rightarrow}\right)=$ $-1,463$ corresponds to adp $+\mathrm{h}+\mathrm{pi} \rightarrow \mathrm{at} \mathrm{p}+\mathrm{h}+\mathrm{h} 2 \mathrm{o}$. Hence, this is the reaction whose educts are most synthesized by other reactions and whose products are the most used as educts in other reactions. It is the bottleneck of the $E$. coli metabolism. Other reactions of this sort, with $F\left(\rightarrow e_{\rightarrow}\right)<-1,000$ (Figure $7 \mathrm{e}$ ), are:

$\mathrm{adp}+\mathrm{h}+\mathrm{pi} \rightarrow \mathrm{atp}+\mathrm{h}+\mathrm{h} 2 \mathrm{o}$

$\mathrm{h}+\mathrm{o} 2+\mathrm{q} 8 \mathrm{~h} 2 \rightarrow \mathrm{h}+\mathrm{h} 2 \mathrm{o}+\mathrm{q} 8$

$\mathrm{h}+\mathrm{o} 2+\mathrm{q} 8 \mathrm{~h} 2 \rightarrow \mathrm{h}+\mathrm{h} 2 \mathrm{o}+\mathrm{q} 8$ $\mathrm{h}+\mathrm{no} 3+\mathrm{q} 8 \mathrm{~h} 2 \rightarrow \mathrm{h}+\mathrm{h} 2 \mathrm{o}+\mathrm{no} 2+\mathrm{q} 8$

$\mathrm{h}+\mathrm{mq} 18+\mathrm{no} 3 \rightarrow \mathrm{h}+\mathrm{h} 2 \mathrm{o}+\mathrm{mqn} 8+\mathrm{no} 2$

Having analyzed the metabolism following the direction of educts to products in reactions, we now proceed to study the curvature in the backward direction, which quantifies to which extent a reaction is just one of the many connecting popular educts with popular products. We start by analyzing $F(\leftarrow e)$ that shows to which extent educts of a reaction participate in other reactions. The theoretical bounds are $-734,375 \leq F(\leftarrow e) \leq 0$ and we found that $F(\leftarrow e)$ takes values in between -729 and 0 ; the minimum is attained by atp $+\mathrm{h}+\mathrm{h} 2 \mathrm{o} \rightarrow \mathrm{adp}+\mathrm{h}+\mathrm{pi}$, indicating that atp in an acidic aqueous medium is the most often used starting material. $\max F(\leftarrow e)$ occurs for 51 reactions, whose involved 56 educts are only used in those 51 reactions, i.e. they are very specialized educts for very particular metabolic reactions. The distribution of $F(\leftarrow e)$ values shows that for half of the reactions, their educts participate in less than $9 \%$ of the reactions, while for the rest, their educts take part in more than $15 \%$ of the reactions.

$F\left(e_{\leftarrow}\right)$ shows to which extent products of a reaction are synthesized by other reactions. The theoretical bounds are given by $\max \left|e_{j}\right|=8$, leading to $-9,400 \leq F\left(e_{\leftarrow}\right) \leq 0$. The actual values range from -788 to 0 . The minimum is reached by reaction: $\mathrm{dxy} 15 \mathrm{p}+\mathrm{nad}+\mathrm{phthr} \rightarrow \mathrm{co} 2+\mathrm{h}+\mathrm{h} 2 \mathrm{o}+\mathrm{nadh}+\mathrm{pdx} 5 \mathrm{p}+\mathrm{pi}$, i.e. this set of products is the most synthesized by $E$. coli metabolism, which is expected, for the likelihood of a set of substances to be synthesized scales with the size of the set. This reaction with six products is one of the few where more than the frequent one to four products are synthesized (Figure 6a). Moreover, among the products, $\mathrm{co} 2, \mathrm{~h}, \mathrm{~h} 2 \mathrm{o}$, nadh, and pi are often products of other reactions.

$\max F\left(e_{\leftarrow}\right)=0$ is attained by 29 reactions, all of them leading to a single product, except for three reactions, each one with two products. Thus, those 32 products are of little synthetic relevance for the metabolism. The distribution of curvature values shows that there are three kinds of reactions whose products are synthesized by different number of reactions. For $60 \%$ of the reactions their products are synthesized by less than 200 reactions $(17 \%$ of the reactions) and for the rest of the reactions by more than 450 reactions ( $38 \%$ of the reactions).

Curvatures $F(\leftarrow e)$ and $F\left(e_{\leftarrow}\right)$ showed that half of the educts are often used and $40 \%$ of the products are often synthesized, which indicates that it is very likely to find alternative ways to link educts with products of existing reactions, as found in [27-29]. A measure of this degree of redundancy of a reaction or of its replaceability is given by $F\left(\leftarrow e_{\leftarrow}\right)$, which indicates to which extent a reaction connects popular educts with popular products. The more negative the curvature, the more redundant or likely replaceable the reaction is.

By analyzing $F\left(\leftarrow e_{\leftarrow}\right)$ distribution (Figure $7 \mathrm{f}$ ) it is seen an ample spectrum of curvatures, with almost no gaps, indicating different degrees of redundancy for the 
metabolic reactions. $\min F\left(\leftarrow e_{\leftarrow}\right)=-1,463$ corresponds to the hydrolysis of ATP, i.e., atp $+\mathrm{h}+\mathrm{h} 2 \mathrm{o} \rightarrow \mathrm{adp}+\mathrm{h}+\mathrm{pi}$, indicating, e.g., that the dephosphorilation of atp to adp can be achieved by many other reactions ( $12 \%$ of the reactions). $\max F\left(\leftarrow e_{\leftarrow}\right)=0$ occurs for the following eight reactions, which are unique as they are the only way to connect their educts with their products:
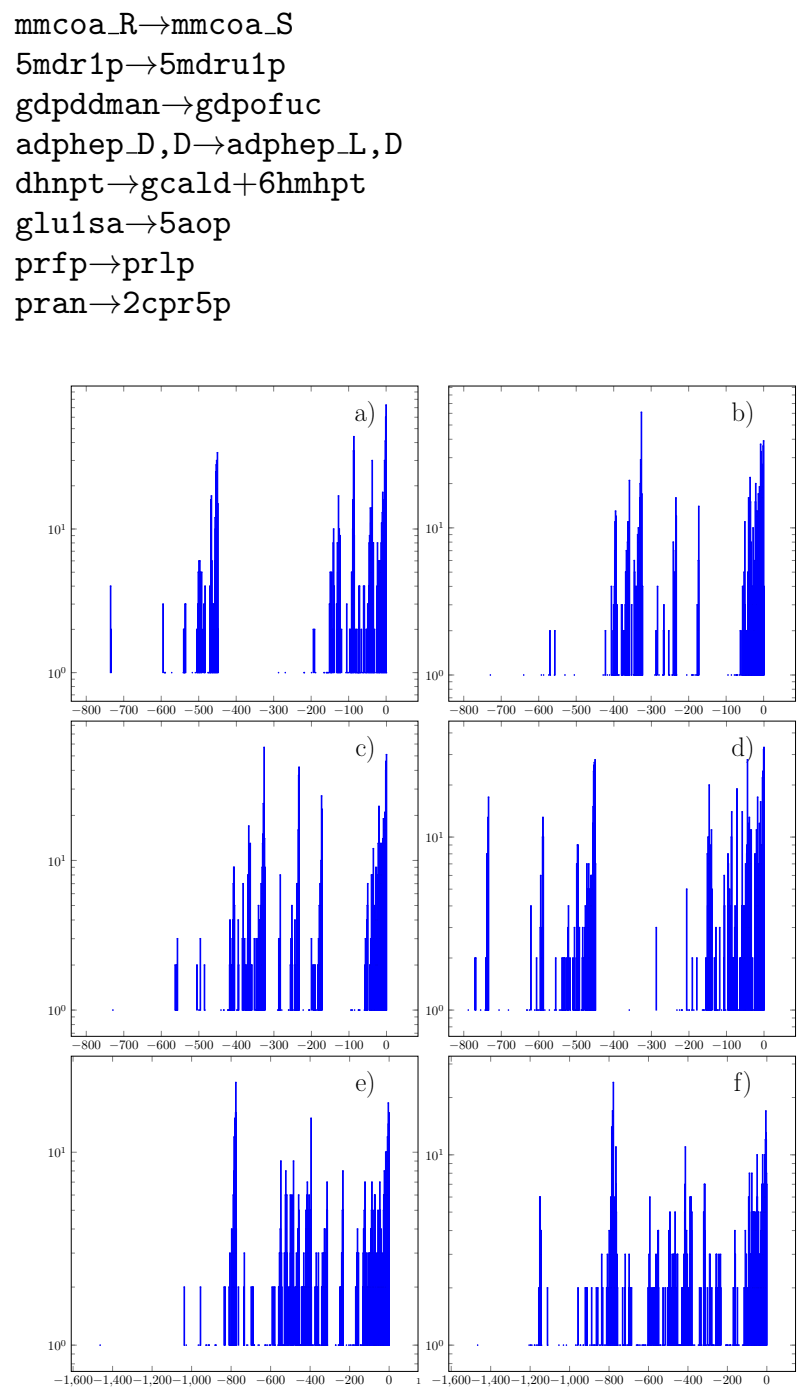

FIG. 7. Metabolic network: Histograms of curvature values for a) $F(\rightarrow e)$, b) $F\left(e_{\rightarrow}\right)$, c) $F(\leftarrow e)$, d) $F\left(e_{\leftarrow}\right)$, e) $F\left(\rightarrow e_{\rightarrow}\right)$, and f) $F\left(\leftarrow e_{\leftarrow}\right)$ with bins of 500 units.

\section{CONCLUSIONS AND OUTLOOK}

The Forman-Ricci curvature emphasizes the importance of the relational character of (hyper)edges, thereby providing a view of the network structure that complements traditional vertex-centered descriptors. It also embeds the characterization in a formal mathematical theory, namely Riemannian geometry.

The results here reported include a brief review of
Forman-Ricci curvature for (un)directed graphs and generalize the curvature to both undirected and directed hypergraphs. Graph curvatures used in previous studies thus become particular cases of the curvature for hypergraphs $[2,11-19]$. We determined the upper and lower bounds for Forman-Ricci curvature for graphs and hypergraphs, which so far had not been studied.

The curvatures here presented aim at quantifying the trade-off between hyperedge(arc) size and the degree of participation of vertices members of the hyperedge $(\operatorname{arc})$ in other hyperedges(arcs). For undirected hypergraphs, the curvature takes negative values when the degree of vertices of the hyperedge is more significant than the size of the hyperedge. For directed hypergraphs we devised four curvatures that gauge different aspects of hyperarcs. $F\left(e_{\rightarrow}\right)$ quantifies the trade-off between the size of the hyperarc tail and the input of its vertices, $F(\rightarrow e)$ do so for the size of the hyperarc head and the output of its vertices; while $F(\leftarrow e)$ and $F\left(e_{\leftarrow}\right)$ consider the size of the tail and the output of their vertices, and the size of the head and the input of their vertices, respectively. These curvatures are combined into $F\left(\rightarrow e_{\rightarrow}\right)$ and $F\left(\leftarrow e_{\leftarrow}\right)$, which account for the flow through hyperarc $e$ and for its redundancy, respectively.

The Forman-Ricci curvature for hypergraphs introduced here differs from the alternative construction proposed in [30]. There, hyperedges are interpreted as simplices. Here, we focus entirely on hyperedges, their sizes, and the degrees of vertices, thereby avoiding the re-interpretation of hyperedges as "higher-dimensional" objects and implicitly introducing additional structures, like boundaries of simplices, that are not part of the original data. For the particular case of directed hypergraphs we disentangled the curvature in the four aforementioned informative measures that allow a detailed exploration of the hypergraph structure. Moreover, we applied these curvatures to the analysis of two large networks, one of social and the other of chemical interactions.

The analysis of Wikipedia vote network exemplified the Forman-Ricci curvature of undirected hypergraphs, where elections constituted hyperedges and users/voters vertices. We found that curvature is mostly ruled by hyperedge size rather than by hyperedge degree. Likewise, the more users involved in elections, the more the presence of experienced users. In a traditional graph setting $[24,25]$, with users as vertices and votes as couple of users, conclusions such as the previous one on elections cannot be drawn. This shows the richness of hypergraphs and their curvatures, which for this particular case allowed the definition of a hyperedge as an election.

Forman-Ricci curvature for directed hypergraphs was computed over the metabolic network of $E$. coli, which traditionally has been analyzed through a graph setting [27-29] and which has shed light on the important role of several substrates for the metabolic stability. In our approach, rather than focusing on substrates, we did on reactions, which were characterized as hyperarcs connecting sets of educts with sets of products. 
In contrast to the Wikipedia vote network, we found that curvature values for the metabolic network were ruled by the degree of hyperarcs, i.e. of in- and outdegrees of tails and heads of hyperarcs. This is a chemical consequence, for it is unlikely that several educts collide simultaneously to give place to a product. In fact reactions where more than five educts participate in a single-step reaction are scarce [31].

We emphasize that the strong dependence of hyperarc curvature is on the summation of the degrees of the vertex belonging to the hyperarc, which is different from the traditional degree of isolated vertices.

With curvature results at hand we defined "bottle neck" reactions as those few reactions whose educts are readily available (obtained from several reactions) and whose products are often used as educts. They are characterized by having very negative $F\left(\rightarrow e_{\rightarrow}\right)$ values. For E. coli this reactions is: adp $+\mathrm{h}+\mathrm{pi} \rightarrow \mathrm{atp}+\mathrm{h}+\mathrm{h} 2$ o. Bottle neck reactions can be considered as assortative ones, for they transform popular products into popular educts.

Curvature values also allowed detecting redundant reactions ("one of the crow reactions"), which can be easily replaced by others. The suitable curvature for detecting such reactions is $F\left(\leftarrow e_{\leftarrow}\right)$, whose most negative values correspond to reactions where popular sets of educts are connected to popular sets of products. For E. coli, this reaction is atp $+\mathrm{h}+\mathrm{h} 2 \mathrm{o} \rightarrow \mathrm{adp}+\mathrm{h}+\mathrm{pi}$. Thus, adp phosphorilation is the metabolic bottle neck reaction but the reverse reaction is not that central for the metabolism.

Our results show that $E$. coli metabolic network makes use of a wealth amount of the products of its reactions to start other reactions. This contrast with the historical trend in wet-lab chemistry reactions, where most of the products are seldom used in further reactions [32]. As the historical study was conducted over single substances, rather than over educts and products, further work on the curvature of wet-lab chemical reactions needs to be done to determine whether the behaviour found for $E$. coli is also a trend of chemical reactions, in general.

The curvatures here presented, as indicated in Equation 1 and as used in [2, 11-19], can be weighted. In the recent sketch of curvature for hypergraphs [30], the weights are calculated based on the volume of the simplex associated to the hyperedge. Weights, however, can also be based on meta information of the network, e.g. user's seniority in the Wikipedia example or stoichiometric coefficients in the metabolic network. This and other weighting schemes need to be explored in future studies on the curvature of hypergraphs, which our approach allows.

\section{ACKNOWLEDGMENTS}

WL was supported by a $\mathrm{PhD}$ scholarship from the German Academic Exchange Service (DAAD): Forschungsstipendien-Promotionen in Deutschland, 2017/2018 (Bewerbung 57299294).

\section{APPENDIX}

[1] A. Bretto, Hypergraph Theory: An Introduction. Springer Publishing Company, Incorporated, 2013.

[2] E. Saucan, A. Samal, M. Weber, and J. Jost, "Discrete curvatures and network analysis," MATCH Communications in Mathematical and in Computer Chemistry, vol. 80, no. 3, pp. 605-622, 2018.

[3] S. Klamt, U.-U. Haus, and F. Theis, "Hypergraphs and cellular networks," PLOS Computational Biology, vol. 5, no. 5, pp. 1-6, 2009.

[4] G. Gallo, G. Longo, S. Pallottino, and S. Nguyen, "Directed hypergraphs and applications," Discrete Applied Mathematics, vol. 42, no. 2, pp. 177 - 201, 1993.

[5] V. C. Barbosa, R. Donangelo, and S. R. Souza, "Coevolution of the mitotic and meiotic modes of eukaryotic cellular division," Phys. Rev. E, vol. 98, p. 032409, 2018.

[6] T. Michoel and B. Nachtergaele, "Alignment and integration of complex networks by hypergraph-based spectral clustering," Phys. Rev. E, vol. 86, p. 056111, 2012.

[7] A. Vazquez, "Population stratification using a statistical model on hypergraphs," Phys. Rev. E, vol. 77, p. 066106, 2008.

[8] F.-L. Xiong, Y.-Z. Zhen, W.-F. Cao, K. Chen, and Z.-B. Chen, "Qudit hypergraph states and their properties," Phys. Rev. A, vol. 97, p. 012323, 2018.
[9] E. Estrada and J. A. Rodríguez-Velázquez, "Subgraph centrality and clustering in complex hyper-networks," Physica A: Statistical Mechanics and its Applications, vol. 364, pp. 581-594, 2006.

[10] B. Schölkopf, J. Platt, and T. Hofmann, Learning with Hypergraphs: Clustering, Classification, and Embedding, pp. 1601-1608. MIT Press, 2007.

[11] A. Samal, R. P. Sreejith, J. Gu, S. Liu, E. Saucan, and J. Jost, "Comparative analysis of two discretizations of Ricci curvature for complex networks," Scientific Reports, vol. 8, 2018.

[12] M. Weber, J. Stelzer, E. Saucan, A. Naitsat, G. Lohmann, and J. Jost, "Curvature-based methods for brain network analysis," ArXiv e-prints, 2017.

[13] E. Saucan and J. Jost, "Network topology vs. geometry : from persistent homology to curvature," Preprint, Max Planck Institute for Mathematics in the Sciences, 2017.

[14] R. P. Sreejith, J. Jost, E. Saucan, and A. Samal, "Systematic evaluation of a new combinatorial curvature for complex networks," Chaos Solitons and Fractals, vol. 101, pp. 50-67, 2017.

[15] M. Weber, E. Saucan, and J. Jost, "Characterizing complex networks with forman-ricci curvature and associated geometric flows," Journal of Complex Networks, vol. 5, no. 4, pp. 527-550, 2017. 
[16] M. Weber, E. Saucan, and J. Jost, "Can one see the shape of a network?," ArXiv e-prints, 2016.

[17] R. P. Sreejith, J. Jost, E. Saucan, and A. Samal, "Forman curvature for directed networks," ArXiv e-prints, 2016.

[18] M. Weber, J. Jost, and E. Saucan, "Forman-ricci flow for change detection in large dynamic data sets," Axioms, vol. 5, p. 26, 2016.

[19] R. P. Sreejith, K. Mohanraj, J. Jost, E. Saucan, and A. Samal, "Forman curvature for complex networks," Journal of Statistical Mechanics: Theory and Experiment, vol. 6, p. 063206, 2016.

[20] F. Bauer, B. Hua, J. Jost, S. Liu, and G. Wang, "The geometric meaning of curvature: Local and nonlocal aspects of ricci curvature," in Modern Approaches to Discrete Curvature (L. Najman and P. Romon, eds.), pp. 1-62, Cham: Springer International Publishing, 2017.

[21] Forman, "Bochner's method for cell complexes and combinatorial Ricci curvature," Discrete \& Computational Geometry, vol. 29, no. 3, pp. 323-374, 2003.

[22] C. Flamm, B. M. R. Stadler, and P. F. Stadler, "Generalized topologies: Hypergreaphs, chemical reactions, and biological evolution," in Advances in Mathematical Chemistry and Applications (S. C. Basak, G. Restrepo, and J. L. Villaveces, eds.), pp. 300-328, BenthamElsevier, 2015.

[23] R. Fagerberg, C. Flamm, R. Kianian, D. Merkle, and P. F. Stadler, "Finding the $k$ best synthesis plans," Journal of Cheminformatics, vol. 10, p. 19, 2018.

[24] "Wikipedia elections network dataset - KONECT," 2017.
[25] J. Leskovec, D. Huttenlocher, and J. Kleinberg, "Governance in social media: A case study of the Wikipedia promotion process," in Proc. Int. Conf. on Weblogs and Social Media, 2010.

[26] J. L. Reed, T. D. Vo, C. H. Schilling, and B. Ø. Palsson, "An expanded genome-scale model of escherichia coli k12 (ijr904 gsm/gpr)," Genome Biol., vol. 4, pp. R54.1R54.12, 2003.

[27] H. Jeong, B. Tombor, R. Albert, Z. N. Oltvai, and A.L. Barabási, "The large-scale organization of metabolic networks," Nature, vol. 407, pp. 651-654, 2000.

[28] A. Wagner and D. A. Fell, "The small world inside large metabolic networks," Proceedings of the Royal Society of London B: Biological Sciences, vol. 268, no. 1478, pp. 1803-1810, 2001.

[29] A.-L. Barabási and R. Albert, "Emergence of scaling in random networks," Science, vol. 286, no. 5439, pp. 509512, 1999.

[30] E. Saucan and M. Weber, "Forman's Ricci curvature - from networks to hypernetworks," Tech. Rep. 1810.07749v1, ArXiv, 2018.

[31] S. Brauch, S. S. van Berkel, and B. Westermann, "Higher-order multicomponent reactions: beyond four reactants," Chem. Soc. Rev., vol. 42, pp. 4948-4962, 2013.

[32] E. J. Llanos, W. Leal, D. H. Luu, J. Jost, P. F. Stadler, and G. Restrepo, "The three historical regimes of the exploration of the chemical space," Submitted to PNAS, 2018. 
TABLE I. Partial list of substrates of the E. coli metabolic network.

Abbreviation Chemical name

\begin{tabular}{|c|c|}
\hline 25aics & $\begin{array}{l}\text { (S)-2-[5-Amino-1-(5-phospho-D- } \\
\text { ribosyl)imidazole- } 4 \text {-carboxamido] succinate }\end{array}$ \\
\hline 5aizc & $\begin{array}{l}\text { 5-amino-1-(5-phospho-D-ribosyl)imidazole-4- } \\
\text { carboxylate }\end{array}$ \\
\hline 5 aop & 5-Amino-4-oxopentanoate \\
\hline $2 c p r 5 p$ & $\begin{array}{l}\text { 1-(2-Carboxyphenylamino)-1-deoxy-D- } \\
\text { ribulose } \\
5 \text {-phosphate }\end{array}$ \\
\hline $6 \mathrm{hmhpt}$ & 6-hydroxymethyl dihydropterin \\
\hline $5 m d r 1 p$ & 5-Methylthio-5-deoxy-D-ribose 1-phosphate \\
\hline 5 mdru1p & 5-Methylthio-5-deoxy-D-ribulose 1-phosphate \\
\hline accoa & Acetyl-CoA \\
\hline adp & Adenosine diphosphate \\
\hline adphep_D,D & ADP-D-glycero-D-manno-heptose \\
\hline adphep_L, D & ADP-L-glycero-D-manno-heptose \\
\hline asp_L & L-Aspartate \\
\hline atp & Adenosine triphosphate \\
\hline $\operatorname{co} 2$ & Carbon dioxyde \\
\hline cyan & Hydrogen cyanide \\
\hline dann & 7,8-Diaminononanoate \\
\hline dhnpt & Dihydroneopterin \\
\hline dtbt & Dethiobiotin \\
\hline dxyl5p & 1-deoxy-D-xylulose 5-phosphate \\
\hline tsul & Thiosulfate \\
\hline gar & N1-(5-Phospho-D-ribosyl)glycinamide \\
\hline gcald & Glycolaldehyde \\
\hline gdpddman & GDP-4-dehydro-6-deoxy-D-mannose \\
\hline gdpofuc & GDP-4-oxo-L-fucose \\
\hline glu1sa & L-Glutamate 1-semialdehyde \\
\hline gly & Glycine \\
\hline h & $\mathrm{H}^{+}$ \\
\hline h2o & Water \\
\hline hco3 & Bicarbonate \\
\hline malcoa & Malonyl CoA C24H33N7O19P3S \\
\hline mmcoa_R & (R)-Methylmalonyl-CoA \\
\hline mmcoa_S & (S)-Methylmalonyl-CoA \\
\hline mql8 & Menaquinol 8 \\
\hline mqn8 & Menaquinone 8 \\
\hline nad & Nicotinamide adenine dinucleotide \\
\hline nadh & Nicotinamide adenine dinucleotide - reduced \\
\hline no2 & Nitrite \\
\hline no3 & Nitrate \\
\hline 02 & Molecular oxygen \\
\hline $\mathrm{pdx} 5 \mathrm{p}$ & Pyridoxine 5'-phosphate \\
\hline phthr & O-Phospho-4-hydroxy-L-threonine \\
\hline pi & Phosphate \\
\hline pram & 5-Phospho-beta-D-ribosylamine \\
\hline pran & N-(5-Phospho-D-ribosyl)anthranilate \\
\hline $\operatorname{prfp}$ & $\begin{array}{l}\text { 1-(5-Phosphoribosyl)-5-[(5- } \\
\text { phosphoribosylamino }) \text { methylideneamino }] \text { imidazole- } \\
\text { 4-carboxamide }\end{array}$ \\
\hline prlp & $\begin{array}{l}\text { 5-[(5-phospho-1-deoxyribulos- } 1- \\
\text { ylamino)methylideneamino]-1- }(5 \text { - } \\
\text { phosphoribosyl }) \text { imidazole-4-carboxamide }\end{array}$ \\
\hline q8 & Ubiquinone- 8 \\
\hline q8h2 & Ubiquinol-8 \\
\hline so3 & Sulfite \\
\hline tcynt & Thiocyanate \\
\hline tsul & Thiosulfate \\
\hline
\end{tabular}




\section{SUPPLEMENTARY MATERIAL}

Curvature values for chemical reactions from the metabolism of Escherichia coli K-12 (iJR904 GSM/GPR).

\begin{tabular}{|c|c|c|c|c|c|c|}
\hline Reaction & $F(\rightarrow e)$ & $F\left(e_{\rightarrow}\right)$ & $F\left(\rightarrow e_{\rightarrow}\right)$ & $F(\leftarrow e)$ & $F\left(e_{\leftarrow}\right)$ & $F\left(\leftarrow e_{\leftarrow}\right)$ \\
\hline ALATA_L $\rightarrow$ & -24 & -42 & -66 & -30 & -77 & -107 \\
\hline ALATA_L $\leftarrow$ & -77 & -30 & -107 & -42 & -24 & -66 \\
\hline $\mathrm{ALAR} \rightarrow$ & -7 & -5 & -12 & -10 & -4 & -14 \\
\hline $\mathrm{ALAR}_{\leftarrow}$ & -4 & -10 & -14 & -5 & -7 & -12 \\
\hline ASNN & -90 & -24 & -114 & -236 & -41 & -277 \\
\hline ASNS2 & -78 & -340 & -418 & -196 & -558 & -754 \\
\hline ASNS1 & -131 & -368 & -499 & -434 & -592 & -1026 \\
\hline ASPT & -7 & -17 & -24 & -17 & -44 & -61 \\
\hline $\mathrm{ASPTA}_{\rightarrow}$ & -24 & -32 & -56 & -37 & -40 & -77 \\
\hline $\mathrm{ASPTA}_{\leftarrow}$ & -40 & -37 & -77 & -32 & -24 & -56 \\
\hline VPAMT & -8 & -17 & -25 & -13 & -47 & -60 \\
\hline DAAD & -91 & -21 & -112 & -245 & -84 & -329 \\
\hline ALARi & -7 & -5 & -12 & -10 & -4 & -14 \\
\hline FFSD & -86 & -6 & -92 & -233 & -8 & -241 \\
\hline A5PISO $\rightarrow$ & -3 & -1 & -4 & -3 & 0 & -3 \\
\hline $\mathrm{A} \mathrm{PISO}_{\leftarrow}$ & 0 & -3 & -3 & -1 & -3 & -4 \\
\hline $\mathrm{MME}_{\rightarrow}$ & -1 & -1 & -2 & 0 & 0 & 0 \\
\hline $\mathrm{MME}_{\leftarrow}$ & 0 & 0 & 0 & -1 & -1 & -2 \\
\hline MICITD & -86 & 0 & -86 & -233 & -1 & -234 \\
\hline ALCD19 $\rightarrow$ & -498 & -55 & -553 & -364 & -45 & -409 \\
\hline ALCD19 & -45 & -364 & -409 & -55 & -498 & -553 \\
\hline LCADi & -130 & -366 & -496 & -287 & -499 & -786 \\
\hline $\mathrm{TGBPA}_{\rightarrow}$ & -1 & -16 & -17 & 0 & -21 & -21 \\
\hline $\mathrm{TGBPA}_{\leftarrow}$ & -21 & 0 & -21 & -16 & -1 & -17 \\
\hline $\mathrm{LCAD}_{\rightarrow}$ & -130 & -366 & -496 & -287 & -499 & -786 \\
\hline $\mathrm{LCAD}_{\leftarrow}$ & -499 & -287 & -786 & -366 & -130 & -496 \\
\hline ALDD $2 x$ & -132 & -365 & -497 & -287 & -508 & -795 \\
\hline $\mathrm{ARAI}_{\rightarrow}$ & -3 & -1 & -4 & -3 & 0 & -3 \\
\hline $\mathrm{ARAI}_{\leftarrow}$ & 0 & -3 & -3 & -1 & -3 & -4 \\
\hline RBK_L1 & -37 & -358 & -395 & -173 & -590 & -763 \\
\hline $\mathrm{RBP}_{4} \mathrm{E}_{\rightarrow}$ & -2 & -3 & -5 & 0 & -4 & -4 \\
\hline $\mathrm{RBP}_{4} \mathrm{E}_{\leftarrow}$ & -4 & 0 & -4 & -3 & -2 & -5 \\
\hline ACACCT & -14 & -3 & -17 & -18 & -14 & -32 \\
\hline BUTCT & -14 & -3 & -17 & -18 & -12 & -30 \\
\hline AB6PGH & -85 & -3 & -88 & -233 & -8 & -241 \\
\hline PMANM $_{\rightarrow}$ & 0 & -2 & -2 & -1 & -3 & -4 \\
\hline PMANM $_{\leftarrow}$ & -3 & -1 & -4 & -2 & 0 & -2 \\
\hline $\mathrm{PPM} 2 \rightarrow$ & -5 & -1 & -6 & -5 & 0 & -5 \\
\hline $\mathrm{PPM} 2_{\leftarrow}$ & 0 & -5 & -5 & -1 & -5 & -6 \\
\hline $\mathrm{PPM}_{\rightarrow}$ & -5 & -3 & -8 & -5 & -8 & -13 \\
\hline $\mathrm{PPM}_{\leftarrow}$ & -8 & -5 & -13 & -3 & -5 & -8 \\
\hline DRPA & 0 & -13 & -13 & -1 & -17 & -18 \\
\hline GALCTND & -1 & -233 & -234 & -2 & -86 & -88 \\
\hline DDPGALA $\rightarrow$ & -1 & -23 & -24 & 0 & -55 & -55 \\
\hline DDPGALA $_{\leftarrow}$ & -55 & 0 & -55 & -23 & -1 & -24 \\
\hline DDGALK & -37 & -358 & -395 & -172 & -589 & -761 \\
\hline DHAPT & -6 & -21 & -27 & -23 & -52 & -75 \\
\hline FA04 & -128 & -362 & -490 & -290 & -505 & -795 \\
\hline ALDD19x & -127 & -362 & -489 & -283 & -496 & -779 \\
\hline FRUK & -37 & -359 & -396 & -172 & -590 & -762 \\
\hline FCLPA $\rightarrow$ & -1 & -11 & -12 & 0 & -12 & -12 \\
\hline $\mathrm{FCLPA}_{\leftarrow}$ & -12 & 0 & -12 & -11 & -1 & -12 \\
\hline $\mathrm{FCI}_{\rightarrow}$ & -2 & -1 & -3 & -2 & 0 & -2 \\
\hline $\mathrm{FCI}_{\leftarrow}$ & 0 & -2 & -2 & -1 & -2 & -3 \\
\hline FCLK & -37 & -358 & -395 & -173 & -589 & -762 \\
\hline $\mathrm{LCAR}_{\rightarrow}$ & -499 & -52 & -551 & -366 & -43 & -409 \\
\hline
\end{tabular}

\begin{tabular}{|c|c|c|c|c|c|c|}
\hline $\mathrm{LCAR}_{\leftarrow}$ & -43 & -366 & -409 & -52 & -499 & -551 \\
\hline UDPG4E $\rightarrow$ & -3 & -2 & -5 & -5 & -1 & -6 \\
\hline $\mathrm{UDPG} \mathrm{E}_{\leftarrow}$ & -1 & -5 & -6 & -2 & -3 & -5 \\
\hline GALKr $\rightarrow$ & -41 & -359 & -400 & -174 & -589 & -763 \\
\hline $\mathrm{GALKr}_{\leftarrow}$ & -589 & -174 & -763 & -359 & -41 & -400 \\
\hline $\mathrm{UGLT}_{\rightarrow}$ & -4 & -11 & -15 & -6 & -7 & -13 \\
\hline $\mathrm{UGLT}_{\leftarrow}$ & -7 & -6 & -13 & -11 & -4 & -15 \\
\hline $\mathrm{GALU}_{\rightarrow}$ & -456 & -12 & -468 & -339 & -75 & -414 \\
\hline GALU $_{\leftarrow}$ & -75 & -339 & -414 & -12 & -456 & -468 \\
\hline GALCTD & -1 & -233 & -234 & -2 & -87 & -89 \\
\hline $\mathrm{GLTPD}_{\rightarrow}$ & -42 & -363 & -405 & -50 & -496 & -546 \\
\hline GLTPD $_{\leftarrow}$ & -496 & -50 & -546 & -363 & -42 & -405 \\
\hline GLYCTO2 & -8 & -6 & -14 & -17 & -18 & -35 \\
\hline GLYCT03 & -11 & -8 & -19 & -13 & -13 & -26 \\
\hline GLYCT04 & -10 & -7 & -17 & -9 & -8 & -17 \\
\hline GLYCDx & -45 & -365 & -410 & -55 & -499 & -554 \\
\hline GLYK & -41 & -365 & -406 & -177 & -597 & -774 \\
\hline PGLYCP & -85 & -40 & -125 & -233 & -151 & -384 \\
\hline G3PD2 $\rightarrow$ & -53 & -374 & -427 & -25 & -475 & -500 \\
\hline G3PD $2 \leftarrow$ & -475 & -25 & -500 & -374 & -53 & -427 \\
\hline GLCRAL & -1 & -15 & -16 & 0 & -45 & -45 \\
\hline DHPPD & -41 & -362 & -403 & -50 & -497 & -547 \\
\hline DHCIND & -41 & -362 & -403 & -50 & -497 & -547 \\
\hline PPPNDO & -501 & -50 & -551 & -384 & -41 & -425 \\
\hline CINNDO & -499 & -50 & -549 & -382 & -41 & -423 \\
\hline $\mathrm{HPYRI}_{\rightarrow}$ & 0 & -1 & -1 & -2 & -2 & -4 \\
\hline HPYRI $_{\leftarrow}$ & -2 & -2 & -4 & -1 & 0 & -1 \\
\hline IDOND $_{\rightarrow}$ & -499 & -52 & -551 & -364 & -46 & -410 \\
\hline IDOND $_{\leftarrow}$ & -46 & -364 & -410 & -52 & -499 & -551 \\
\hline IDOND2 & -469 & -20 & -489 & -369 & -49 & -418 \\
\hline GNK & -42 & -359 & -401 & -175 & -589 & -764 \\
\hline 5DGLCNR $\rightarrow$ & -469 & -21 & -490 & -369 & -49 & -418 \\
\hline $5 \mathrm{DGLCNR}_{\leftarrow}$ & -49 & -369 & -418 & -21 & -469 & -490 \\
\hline DDGLK & -40 & -358 & -398 & -174 & -589 & -763 \\
\hline LACZ & -87 & -5 & -92 & -235 & -20 & -255 \\
\hline MLTP1 $\rightarrow$ & -150 & -12 & -162 & -40 & -9 & -49 \\
\hline MLTP1 $1_{\leftarrow}$ & -9 & -40 & -49 & -12 & -150 & -162 \\
\hline MLTP2 $\rightarrow$ & -150 & -13 & -163 & -40 & -10 & -50 \\
\hline MLTP $2_{\leftarrow}$ & -10 & -40 & -50 & -13 & -150 & -163 \\
\hline MLTP3 $\rightarrow$ & -147 & -13 & -160 & -37 & -10 & -47 \\
\hline MLTP3 $_{\leftarrow}$ & -10 & -37 & -47 & -13 & -147 & -160 \\
\hline AMALT1 & -2 & -6 & -8 & -7 & -19 & -26 \\
\hline AMALT2 & -4 & -7 & -11 & -8 & -20 & -28 \\
\hline AMALT3 & -5 & -7 & -12 & -9 & -20 & -29 \\
\hline AMALT4 & -5 & -4 & -9 & -9 & -17 & -26 \\
\hline MLTG1 & -87 & -8 & -95 & -235 & -17 & -252 \\
\hline MLTG2 & -89 & -5 & -94 & -236 & -17 & -253 \\
\hline MLTG3 & -90 & -6 & -96 & -237 & -19 & -256 \\
\hline MLTG4 & -90 & -7 & -97 & -237 & -20 & -257 \\
\hline MLTG5 & -87 & -7 & -94 & -234 & -20 & -254 \\
\hline MAN6PI $\rightarrow_{\rightarrow}$ & -3 & -8 & -11 & -2 & -9 & -11 \\
\hline MAN6PI $_{\leftarrow}$ & -9 & -2 & -11 & -8 & -3 & -11 \\
\hline GALS3 & -86 & -5 & -91 & -234 & -20 & -254 \\
\hline 3HCINNMH & -501 & -283 & -784 & -384 & -128 & -512 \\
\hline 3HPPPNH & -501 & -283 & -784 & -384 & -128 & -512 \\
\hline DHCINDO & -5 & 0 & -5 & -20 & 0 & -20 \\
\hline HPPPNDO & -5 & 0 & -5 & -20 & 0 & -20 \\
\hline HKNDDH & -86 & -336 & -422 & -233 & -471 & -704 \\
\hline HKNTDH & -86 & -334 & -420 & -233 & -460 & -693 \\
\hline OP4ENH & -87 & 0 & -87 & -233 & 0 & -233 \\
\hline HOPNTAL & 0 & -18 & -18 & 0 & -48 & -48 \\
\hline ACALDi & -69 & -378 & -447 & -75 & -509 & -584 \\
\hline $\mathrm{M} 1 \mathrm{PD}_{\rightarrow}$ & -42 & -370 & -412 & -50 & -505 & -555 \\
\hline $\mathrm{M} 1 \mathrm{PD}_{\leftarrow}$ & -505 & -50 & -555 & -370 & -42 & -412 \\
\hline
\end{tabular}




\begin{tabular}{|c|c|c|c|c|c|c|c|c|c|c|c|c|c|}
\hline AGDC & -87 & -4 & -91 & -233 & -15 & -248 & ME2 & -48 & -65 & -113 & -25 & -118 & -143 \\
\hline G6PDA & -89 & -15 & -104 & -234 & -43 & -277 & PPCK & -43 & -62 & -105 & -176 & -200 & -376 \\
\hline ACNML & 0 & -15 & -15 & -1 & -43 & -44 & PPA & -158 & -360 & -518 & -240 & -595 & -835 \\
\hline TRE6PS & -11 & -327 & -338 & -9 & -456 & -465 & PPC & -147 & -364 & -511 & -261 & -601 & -862 \\
\hline TRE6PP & -87 & -38 & -125 & -234 & -146 & -380 & ME1 & -45 & -60 & -105 & -57 & -148 & -205 \\
\hline PACCOAL & -60 & -12 & -72 & -193 & -105 & -298 & MDRPD & 0 & -234 & -234 & -1 & -86 & -87 \\
\hline PFK_2 & -37 & -358 & -395 & -173 & -589 & -762 & DKMPPD2 & -86 & -365 & -451 & -234 & -606 & -840 \\
\hline $\mathrm{PGMT} \rightarrow$ & -6 & -4 & -10 & -9 & -8 & -17 & PTRCTA & -23 & -28 & -51 & -26 & -34 & -60 \\
\hline $\mathrm{PGMT}_{\leftarrow}$ & -8 & -9 & -17 & -4 & -6 & -10 & SSALX & -127 & -374 & -501 & -284 & -517 & -801 \\
\hline MCITL2 $\rightarrow$ & -1 & -26 & -27 & 0 & -64 & -64 & ABUTD & -127 & -365 & -492 & -283 & -500 & -783 \\
\hline MCITL $2_{\leftarrow}$ & -64 & 0 & -64 & -26 & -1 & -27 & MTRK & -37 & -358 & -395 & -172 & -589 & -761 \\
\hline MCITS & -94 & -345 & -439 & -239 & -472 & -711 & $\mathrm{MTRI}_{\rightarrow}$ & -1 & -1 & -2 & 0 & 0 & 0 \\
\hline MCITD & 0 & -233 & -233 & 0 & -86 & -86 & $\mathrm{MTRI}_{\leftarrow}$ & 0 & 0 & 0 & -1 & -1 & -2 \\
\hline ACCOAL & -61 & -72 & -133 & -194 & -287 & -481 & DKMPPD & -90 & -365 & -455 & -254 & -606 & -860 \\
\hline PTA2 & -148 & -21 & -169 & -38 & -24 & -62 & G5SADs & -2 & -558 & -560 & 0 & -536 & -536 \\
\hline $\mathrm{RBK}$ & -37 & -361 & -398 & -173 & -596 & -769 & UNK3 & -35 & -21 & -56 & -28 & -19 & -47 \\
\hline $\mathrm{RMI}_{\rightarrow}$ & -1 & -1 & -2 & -1 & 0 & -1 & ACGS & -47 & -345 & -392 & -44 & -472 & -516 \\
\hline $\mathrm{RMI}_{\leftarrow}$ & 0 & -1 & -1 & -1 & -1 & -2 & ACGK & -37 & -34 & -71 & -172 & -140 & -312 \\
\hline RMK & -37 & -358 & -395 & -173 & -589 & -762 & $\mathrm{AGPR}_{\rightarrow}$ & -191 & -367 & -558 & -56 & -467 & -523 \\
\hline $\mathrm{RMPA}_{\rightarrow}$ & -1 & -11 & -12 & 0 & -12 & -12 & $\mathrm{AGPR}_{\leftarrow}$ & -467 & -56 & -523 & -367 & -191 & -558 \\
\hline $\mathrm{RMPA}_{\leftarrow}$ & -12 & 0 & -12 & -11 & -1 & -12 & $\mathrm{ACOTA}_{\rightarrow}$ & -17 & -30 & -47 & -21 & -35 & -56 \\
\hline MMM2 & -2 & 0 & -2 & -4 & -1 & -5 & $\mathrm{ACOTA}_{\leftarrow}$ & -35 & -21 & -56 & -30 & -17 & -47 \\
\hline KG6PDC & -448 & -8 & -456 & -324 & -58 & -382 & NACODA & -87 & -3 & -90 & -235 & -14 & -249 \\
\hline X5PL3E & 0 & 0 & 0 & 0 & -2 & -2 & ACODA & -86 & -10 & -96 & -234 & -18 & -252 \\
\hline $\mathrm{SBTPD} \rightarrow$ & -42 & -370 & -412 & -50 & -505 & -555 & ARGSS & -44 & -337 & -381 & -190 & -555 & -745 \\
\hline $\mathrm{SBTPD}_{\leftarrow}$ & -505 & -50 & -555 & -370 & -42 & -412 & $\mathrm{ARGSL}_{\rightarrow}$ & -1 & -15 & -16 & 0 & -13 & -13 \\
\hline TAUDO & -21 & -343 & -364 & -41 & -531 & -572 & $\mathrm{ARGSL}_{\leftarrow}$ & -13 & 0 & -13 & -15 & -1 & -16 \\
\hline $\mathrm{PPAKr} \rightarrow$ & -140 & -173 & -313 & -34 & -38 & -72 & $\mathrm{OCBT}_{\rightarrow}$ & -8 & -361 & -369 & -8 & -595 & -603 \\
\hline $\mathrm{PPAKr}_{\leftarrow}$ & -38 & -34 & -72 & -173 & -140 & -313 & $\mathrm{OCBT}_{\leftarrow}$ & -595 & -8 & -603 & -361 & -8 & -369 \\
\hline OBTFL & -23 & -7 & -30 & -22 & -12 & -34 & AST & -5 & -345 & -350 & -9 & -472 & -481 \\
\hline TREHe & -86 & -3 & -89 & -235 & -16 & -251 & SOTA & -17 & -28 & -45 & -20 & -34 & -54 \\
\hline TRE6PH & -87 & -7 & -94 & -234 & -24 & -258 & SADH & -535 & -15 & -550 & -557 & -92 & -649 \\
\hline TREH & -86 & -3 & -89 & -235 & -16 & -251 & SGSAD & -127 & -362 & -489 & -283 & -496 & -779 \\
\hline TARTD & -1 & -237 & -238 & -2 & -92 & -94 & SGDS & -86 & -40 & -126 & -233 & -55 & -288 \\
\hline PEAMNO & -89 & -8 & -97 & -253 & -38 & -291 & CBPS & -125 & -423 & -548 & -421 & -770 & -1191 \\
\hline ALTRH & 0 & -235 & -235 & -1 & -89 & -90 & SSALy & -130 & -379 & -509 & -252 & -487 & -739 \\
\hline TAGURr $\rightarrow$ & -41 & -363 & -404 & -51 & -497 & -548 & ABTA & -21 & -29 & -50 & -23 & -34 & -57 \\
\hline TAGUR $_{\leftarrow}$ & -497 & -51 & -548 & -363 & -41 & -404 & GSPMDA & -86 & -5 & -91 & -233 & -5 & -238 \\
\hline GUI1 $\rightarrow$ & -2 & -1 & -3 & -2 & -1 & -3 & GSPMDS & -42 & -394 & -436 & -177 & -734 & -911 \\
\hline GUI1 $\leftarrow$ & -1 & -2 & -3 & -1 & -2 & -3 & MTAN & -86 & -5 & -91 & -233 & -6 & -239 \\
\hline GUI2 $\rightarrow$ & -2 & -1 & -3 & -2 & -1 & -3 & G5SD & -466 & -54 & -520 & -367 & -192 & -559 \\
\hline GUI2 $_{\leftarrow}$ & -1 & -2 & -3 & -1 & -2 & -3 & GLU5K & -71 & -34 & -105 & -200 & -139 & -339 \\
\hline MNNH & 0 & -235 & -235 & -1 & -89 & -90 & P5CR & -467 & -22 & -489 & -368 & -48 & -416 \\
\hline MANAO $\rightarrow$ & -41 & -363 & -404 & -51 & -497 & -548 & P5CD & -128 & -390 & -518 & -284 & -530 & -814 \\
\hline $\mathrm{MANAO}_{\leftarrow}$ & -497 & -51 & -548 & -363 & -41 & -404 & PROD2 & -5 & -325 & -330 & -11 & -457 & -468 \\
\hline XYLI2i & 0 & -3 & -3 & -2 & -16 & -18 & ARGDC & -452 & -8 & -460 & -329 & -58 & -387 \\
\hline XYLI1 $\rightarrow$ & -2 & -1 & -3 & -2 & 0 & -2 & AGMT & -86 & -7 & -93 & -233 & -9 & -242 \\
\hline XYLI1 $\leftarrow$ & 0 & -2 & -2 & -1 & -2 & -3 & ORNDC & -455 & -14 & -469 & -331 & -64 & -395 \\
\hline XYLK & -37 & -361 & -398 & -173 & -592 & -765 & $\mathrm{ADMDCr} \rightarrow$ & -451 & -9 & -460 & -331 & -58 & -389 \\
\hline DKGLCNR1 & -465 & -19 & -484 & -369 & -44 & -413 & $\mathrm{ADMDCr}_{\leftarrow}$ & -58 & -331 & -389 & -9 & -451 & -460 \\
\hline HPYRRx & -496 & -50 & -546 & -364 & -43 & -407 & SPMS & -6 & -327 & -333 & -7 & -451 & -458 \\
\hline HPYRRy & -466 & -18 & -484 & -369 & -46 & -415 & SPMDAT1 & -15 & -344 & -359 & -19 & -472 & -491 \\
\hline GLCRD & -1 & -233 & -234 & -2 & -87 & -89 & SPMDAT2 & -15 & -344 & -359 & -19 & -472 & -491 \\
\hline MMCD & -449 & -10 & -459 & -325 & -60 & -385 & ORNTA & -23 & -28 & -51 & -27 & -36 & -63 \\
\hline PPCSCT & -23 & -5 & -28 & -14 & -3 & -17 & PPTGS & 0 & -323 & -323 & 0 & -451 & -451 \\
\hline DKGLCNR2y & -465 & -20 & -485 & -369 & -47 & -416 & UDCPDP & -88 & -361 & -449 & -233 & -595 & -828 \\
\hline 2DGLCNRx & -495 & -53 & -548 & -363 & -46 & -409 & KDOPP & -86 & -36 & -122 & -233 & -146 & -379 \\
\hline 2DGLCNRy & -465 & -21 & -486 & -368 & -49 & -417 & PEPT_EC $\rightarrow$ & -461 & -2 & -463 & -330 & -1 & -331 \\
\hline DKGLCNR2x & -495 & -52 & -547 & -364 & -44 & -408 & $\mathrm{PEPT}_{-} \mathrm{EC}_{\leftarrow}$ & -1 & -330 & -331 & -2 & -461 & -463 \\
\hline 2DGULRx & -496 & -52 & -548 & -363 & -46 & -409 & PAPA_EC & -89 & -37 & -126 & -234 & -147 & -381 \\
\hline 2DGULRy & -466 & -20 & -486 & -368 & -49 & -417 & ECAP_EC & 0 & -323 & -323 & 0 & -451 & -451 \\
\hline DOGULNR & -495 & -49 & -544 & -362 & -41 & -403 & AACPS1 & -60 & -15 & -75 & -181 & -106 & -287 \\
\hline ICL & -1 & -15 & -16 & -2 & -25 & -27 & AACPS2 & -59 & -13 & -72 & -179 & -106 & -285 \\
\hline MALS & -103 & -352 & -455 & -252 & -476 & -728 & AACPS3 & -60 & -13 & -73 & -181 & -106 & -287 \\
\hline
\end{tabular}




\begin{tabular}{|c|c|c|c|c|c|c|c|c|c|c|c|c|c|}
\hline AACPS4 & -59 & -14 & -73 & -179 & -106 & -285 & TDPDRR & -466 & -17 & -483 & -367 & -44 & -411 \\
\hline AACPS5 & -59 & -13 & -72 & -179 & -106 & -285 & MI1PP & -85 & -35 & -120 & -233 & -147 & -380 \\
\hline ALAALAr $_{\rightarrow}$ & -41 & -395 & -436 & -177 & -734 & -911 & UDPGD & -130 & -361 & -491 & -288 & -496 & -784 \\
\hline ALAALAr $_{\leftarrow}$ & -734 & -177 & -911 & -395 & -41 & -436 & USHD & -86 & -325 & -411 & -234 & -456 & -690 \\
\hline DAGK_EC & -38 & -359 & -397 & -173 & -591 & -764 & ACGAMT & -1 & -1 & -2 & -5 & -7 & -12 \\
\hline ETHAAL & 0 & -11 & -11 & 0 & -39 & -39 & UAG2Ei & -1 & 0 & -1 & -4 & 0 & -4 \\
\hline GDMANE & 0 & 0 & 0 & 0 & 0 & 0 & UACMAMO & -127 & -362 & -489 & -283 & -496 & -779 \\
\hline GOFUCR & -466 & -17 & -483 & -367 & -44 & -411 & TDPADGAT & -13 & -345 & -358 & -16 & -472 & -488 \\
\hline GALUi & -456 & -12 & -468 & -339 & -75 & -414 & TDPAGTA & -34 & -20 & -54 & -29 & -17 & -46 \\
\hline UDPGALM & -1 & 1 & 0 & -2 & 0 & -2 & AADDGT & 0 & -325 & -325 & 0 & -451 & -451 \\
\hline GF6PTA & -10 & -29 & -39 & -20 & -37 & -57 & ACMAMUT & 0 & -326 & -326 & 0 & -455 & -455 \\
\hline UAGDP & -450 & -11 & -461 & -330 & -73 & -403 & $\mathrm{ACONT}_{\rightarrow}$ & -2 & -2 & -4 & -2 & -1 & -3 \\
\hline G1PACT & -13 & -345 & -358 & -17 & -472 & -489 & $\mathrm{ACONT}_{\leftarrow}$ & -1 & -2 & -3 & -2 & -2 & -4 \\
\hline GPDDA1 & -87 & -333 & -420 & -233 & -461 & -694 & CITL & -2 & -7 & -9 & -2 & -18 & -20 \\
\hline GPDDA2 & -87 & -331 & -418 & -233 & -458 & -691 & FRD2 & -19 & -21 & -40 & -15 & -27 & -42 \\
\hline GPDDA3 & -85 & -340 & -425 & -233 & -465 & -698 & FRD3 & -14 & -17 & -31 & -14 & -26 & -40 \\
\hline GPDDA4 & -87 & -336 & -423 & -233 & -462 & -695 & $\mathrm{FUM}_{\rightarrow}$ & -96 & -7 & -103 & -243 & -4 & -247 \\
\hline GPDDA5 & -85 & -330 & -415 & -233 & -459 & -692 & FUM $_{\leftarrow}$ & -4 & -243 & -247 & -7 & -96 & -103 \\
\hline GMAND & 0 & -233 & -233 & 0 & -86 & -86 & CS & -105 & -347 & -452 & -253 & -474 & -727 \\
\hline S7PI & -1 & 0 & -1 & -2 & 0 & -2 & $\mathrm{ICDHyr}_{\rightarrow}$ & -45 & -71 & -116 & -20 & -92 & -112 \\
\hline GMHEPPA & -86 & -36 & -122 & -233 & -146 & -379 & ICDHyr $_{\leftarrow}$ & -92 & -20 & -112 & -71 & -45 & -116 \\
\hline KDOPS & -89 & -36 & -125 & -254 & -146 & -400 & TEST_AKGDH & -81 & -50 & -131 & -91 & -107 & -198 \\
\hline KDOCT2 & -2 & -9 & -11 & -6 & -72 & -78 & $\mathrm{MDH}_{\rightarrow}$ & -45 & -366 & -411 & -57 & -502 & -559 \\
\hline MOAT & 0 & -329 & -329 & -2 & -460 & -462 & $\mathrm{MDH}_{\leftarrow}$ & -502 & -57 & -559 & -366 & -45 & -411 \\
\hline MOAT2 & 0 & -330 & -330 & -2 & -460 & -462 & MDH2 & -7 & -7 & -14 & -20 & -20 & -40 \\
\hline $\mathrm{UAGAAT}_{\rightarrow}$ & -2 & -8 & -10 & -5 & -17 & -22 & MDH3 & -10 & -9 & -19 & -16 & -15 & -31 \\
\hline $\mathrm{UAGAAT}_{\leftarrow}$ & -17 & -5 & -22 & -8 & -2 & -10 & SUCD1i & -22 & -10 & -32 & -19 & -17 & -36 \\
\hline LPADSS & 0 & -326 & -326 & -1 & -455 & -456 & $\mathrm{SUCOAS}_{\rightarrow}$ & -81 & -74 & -155 & -205 & -287 & -492 \\
\hline UHGADA & -86 & -3 & -89 & -234 & -12 & -246 & SUCOAS $_{\leftarrow}$ & -287 & -205 & -492 & -74 & -81 & -155 \\
\hline U23GAAT & -1 & -332 & -333 & -1 & -466 & -467 & PMDPHT & -86 & -36 & -122 & -233 & -147 & -380 \\
\hline TDSK & -37 & -358 & -395 & -172 & -588 & -760 & DNMPPA & -86 & -36 & -122 & -233 & -146 & -379 \\
\hline EDTXS1 & 0 & -7 & -7 & -2 & -17 & -19 & NADPPPS & -130 & -86 & -216 & -251 & -187 & -438 \\
\hline EDTXS3 & -1 & -7 & -8 & -2 & -17 & -19 & NMNN & -88 & -327 & -415 & -237 & -458 & -695 \\
\hline MAN1PT2 & -456 & -36 & -492 & -329 & -146 & -475 & NADDPe & -127 & -334 & -461 & -283 & -484 & -767 \\
\hline РАРPТЗ & 0 & -1 & -1 & -1 & -7 & -8 & PPCDC & -449 & -8 & -457 & -324 & -58 & -382 \\
\hline $\mathrm{PGAMT}_{\rightarrow}$ & 0 & -1 & -1 & -1 & -3 & -4 & DPCOAK & -37 & -379 & -416 & -172 & -611 & -783 \\
\hline $\mathrm{PGAMT}_{\leftarrow}$ & -3 & -1 & -4 & -1 & 0 & -1 & HYPOE & -88 & -36 & -124 & -234 & -146 & -380 \\
\hline EDTXS4 & -1 & -6 & -7 & -2 & -17 & -19 & PYDXPP & -88 & -36 & -124 & -236 & -146 & -382 \\
\hline EDTXS2 & -1 & -7 & -8 & -2 & -17 & -19 & PDXPP & -88 & -36 & -124 & -234 & -146 & -380 \\
\hline UAGCVT & -4 & -36 & -40 & -24 & -146 & -170 & NMNDA & -88 & -8 & -96 & -237 & -36 & -273 \\
\hline UAPGR & -466 & -18 & -484 & -367 & -44 & -411 & $\mathrm{TMKr}_{\rightarrow}$ & -38 & -359 & -397 & -173 & -590 & -763 \\
\hline UAMAS & -44 & -394 & -438 & -182 & -734 & -916 & $\mathrm{TMKr}_{\leftarrow}$ & -590 & -173 & -763 & -359 & -38 & -397 \\
\hline UAMAGS & -37 & -394 & -431 & -173 & -734 & -907 & PPNCL2 & -4 & -336 & -340 & -12 & -532 & -544 \\
\hline UAAGDS & -38 & -394 & -432 & -175 & -734 & -909 & ACPS1 & -22 & -331 & -353 & -21 & -467 & -488 \\
\hline UGMDDS & -37 & -394 & -431 & -173 & -734 & -907 & $\mathrm{AMAOTr}_{\rightarrow}$ & -3 & -1 & -4 & -8 & -1 & -9 \\
\hline UAGPT3 & -1 & -326 & -327 & -4 & -455 & -459 & $\mathrm{AMAOTr}_{\leftarrow}$ & -1 & -8 & -9 & -1 & -3 & -4 \\
\hline GLUR $\rightarrow$ & 0 & -28 & -28 & -1 & -34 & -35 & BTS2 $\rightarrow$ & -3 & -334 & -337 & -7 & -458 & -465 \\
\hline GLUR $_{\leftarrow}$ & -34 & -1 & -35 & -28 & 0 & -28 & $\mathrm{BTS} 2_{\leftarrow}$ & -458 & -7 & -465 & -334 & -3 & -337 \\
\hline PLIPA3 & -85 & -329 & -414 & -233 & -476 & -709 & $\mathrm{DBTSr}_{\rightarrow}$ & -96 & -395 & -491 & -181 & -735 & -916 \\
\hline PLIPA1 & -87 & -329 & -416 & -237 & -476 & -713 & $\mathrm{DBTSr}_{\leftarrow}$ & -735 & -181 & -916 & -395 & -96 & -491 \\
\hline PLIPA2 & -87 & -329 & -416 & -234 & -476 & -710 & $\mathrm{AOXSr}_{\rightarrow}$ & -456 & -30 & -486 & -334 & -82 & -416 \\
\hline LPLIPA1 & -86 & -328 & -414 & -234 & -477 & -711 & $\mathrm{AOXSr}_{\leftarrow}$ & -82 & -334 & -416 & -30 & -456 & -486 \\
\hline LPLIPA2 & -86 & -328 & -414 & -234 & -477 & -711 & BSORx & -495 & -283 & -778 & -363 & -129 & -492 \\
\hline LPLIPA3 & -86 & -328 & -414 & -234 & -477 & -711 & BSORy & -465 & -251 & -716 & -368 & -132 & -500 \\
\hline LPLIPA4 & -1 & 1 & 0 & -5 & -3 & -8 & $\mathrm{CBIAT}_{\rightarrow}$ & -123 & -44 & -167 & -405 & -218 & -623 \\
\hline LPLIPA5 & -1 & 1 & 0 & -5 & -3 & -8 & $\mathrm{CBIAT}_{\leftarrow}$ & -218 & -405 & -623 & -44 & -123 & -167 \\
\hline LPLIPA6 & -1 & 1 & 0 & -5 & -3 & -8 & $\mathrm{CBLAT}_{\rightarrow}$ & -124 & -43 & -167 & -406 & -219 & -625 \\
\hline AGMHE & 0 & 0 & 0 & 0 & 0 & 0 & $\mathrm{CBLAT}_{\leftarrow}$ & -219 & -406 & -625 & -43 & -124 & -167 \\
\hline GMHEPAT & -486 & -7 & -493 & -496 & -72 & -568 & PNTK & -38 & -358 & -396 & -173 & -588 & -761 \\
\hline GMHEPK & -37 & -358 & -395 & -172 & -588 & -760 & PTPATi & -486 & -7 & -493 & -496 & -72 & -568 \\
\hline LPSSYN_EC & -3 & -366 & -369 & -8 & -607 & -615 & ADOCBLS & 0 & -326 & -326 & 0 & -455 & -455 \\
\hline G1PTT & -455 & -7 & -462 & -335 & -72 & -407 & NNDMBRT & -1 & -325 & -326 & -1 & -451 & -452 \\
\hline TDPGDH & 0 & -234 & -234 & 0 & -86 & -86 & ADOCBIK & -37 & -358 & -395 & -173 & -588 & -761 \\
\hline TDPDRE & 0 & 0 & 0 & -1 & 0 & -1 & ACBIPGT & -450 & -7 & -457 & -335 & -72 & -407 \\
\hline
\end{tabular}




\begin{tabular}{|c|c|c|c|c|c|c|c|c|c|c|c|c|c|}
\hline HEMEOS & -86 & -6 & -92 & -235 & -72 & -307 & NNAT & -488 & -7 & -495 & -497 & -72 & -569 \\
\hline SHCHD2 & -41 & -362 & -403 & -50 & -496 & -546 & NMNAT & -488 & -57 & -545 & -500 & -113 & -613 \\
\hline SHCHF & 0 & -323 & -323 & -2 & -449 & -451 & NADS1 & -71 & -387 & -458 & -179 & -595 & -774 \\
\hline DXPRIi & -467 & -18 & -485 & -369 & -44 & -413 & DNTPPA & -86 & -331 & -417 & -233 & -521 & -754 \\
\hline DXPS & -504 & -10 & -514 & -347 & -59 & -406 & ADCS & -1 & -28 & -29 & -16 & -34 & -50 \\
\hline $\mathrm{DHBD}_{\rightarrow}$ & -42 & -363 & -405 & -50 & -497 & -547 & ADCL & 0 & -338 & -338 & 0 & -492 & -492 \\
\hline $\mathrm{DHBD}_{\leftarrow}$ & -497 & -50 & -547 & -363 & -42 & -405 & МОНМТ & -89 & -4 & -93 & -239 & -5 & -244 \\
\hline ICHORT & -86 & -14 & -100 & -234 & -44 & -278 & PANTS & -37 & -338 & -375 & -172 & -555 & -727 \\
\hline ENTCS & 0 & -329 & -329 & -2 & -482 & -484 & ASP1DC & -456 & -8 & -464 & -341 & -58 & -399 \\
\hline $\mathrm{DHBSr}_{\rightarrow}$ & -38 & -8 & -46 & -173 & -72 & -245 & DPR & -466 & -18 & -484 & -367 & -44 & -411 \\
\hline $\mathrm{DHBSr}_{\leftarrow}$ & -72 & -173 & -245 & -8 & -38 & -46 & PDX5PS & -42 & -640 & -682 & -54 & -788 & -842 \\
\hline SERASr $\rightarrow$ & -493 & -8 & -501 & -505 & -72 & -577 & $\mathrm{PERD}_{\rightarrow}$ & -42 & -363 & -405 & -51 & -497 & -548 \\
\hline $\mathrm{SERASr}_{\leftarrow}$ & -72 & -505 & -577 & -8 & -493 & -501 & $\mathrm{PERD}_{\leftarrow}$ & -497 & -51 & -548 & -363 & -42 & -405 \\
\hline $\mathrm{E} 4 \mathrm{PD} \rightarrow$ & -129 & -363 & -492 & -286 & -497 & -783 & $\mathrm{PDX} 5 \mathrm{PO} \rightarrow$ & -6 & -4 & -10 & -21 & -6 & -27 \\
\hline $\mathrm{E} 4 \mathrm{PD}_{\leftarrow}$ & -497 & -286 & -783 & -363 & -129 & -492 & $\mathrm{PDX}_{5 \mathrm{PO}} \leftarrow$ & -6 & -21 & -27 & -4 & -6 & -10 \\
\hline $\mathrm{DHFR}_{\rightarrow}$ & -468 & -22 & -490 & -367 & -49 & -416 & PYAM5PO & -92 & -11 & -103 & -254 & -40 & -294 \\
\hline $\mathrm{DHFR}_{\leftarrow}$ & -49 & -367 & -416 & -22 & -468 & -490 & PYDXNK & -37 & -359 & -396 & -172 & -590 & -762 \\
\hline DHNPA2 & 0 & 0 & 0 & 0 & 0 & 0 & PYDAMK & -37 & -359 & -396 & -172 & -590 & -762 \\
\hline DHFS & -71 & -70 & -141 & -200 & -287 & -487 & PYDXK & -37 & -361 & -398 & -172 & -590 & -762 \\
\hline GTPCI & -87 & -5 & -92 & -244 & -10 & -254 & RZ5PP & -86 & -36 & -122 & -233 & -146 & -379 \\
\hline HPPK2 & -37 & -330 & -367 & -172 & -482 & -654 & NNAM & -87 & -8 & -95 & -233 & -36 & -269 \\
\hline DHPS2 & 0 & -331 & -331 & 0 & -521 & -521 & NAMNPP & -126 & -78 & -204 & -417 & -359 & -776 \\
\hline MECDPDH & -449 & -234 & -683 & -324 & -86 & -410 & GTPCII2 & -87 & -336 & -423 & -244 & -531 & -775 \\
\hline GLUTRS & -71 & -13 & -84 & -200 & -105 & -305 & DB4PS & -3 & -329 & -332 & -3 & -459 & -462 \\
\hline ALATA_D2 & -6 & -15 & -21 & -8 & -45 & -53 & APRAUR & -466 & -18 & -484 & -367 & -44 & -411 \\
\hline ALATA_L2 & -9 & -15 & -24 & -13 & -45 & -58 & DHPPDA2 & -535 & -7 & -542 & -557 & -34 & -591 \\
\hline GTHOr $_{\rightarrow}$ & -466 & -20 & -486 & -367 & -47 & -414 & RBFSa & -1 & -269 & -270 & 0 & -232 & -232 \\
\hline $\mathrm{GTHOr}_{\leftarrow}$ & -47 & -367 & -414 & -20 & -466 & -486 & RBFK & -37 & -358 & -395 & -172 & -588 & -760 \\
\hline GLUCYS & -73 & -394 & -467 & -206 & -734 & -940 & FMNAT & -486 & -14 & -500 & -496 & -73 & -569 \\
\hline GTHS & -42 & -396 & -438 & -177 & -737 & -914 & RBFSb & 0 & 0 & 0 & 0 & -1 & -1 \\
\hline GLUTRR & -466 & -18 & -484 & -367 & -44 & -411 & $\mathrm{OHPBAT}_{\rightarrow}$ & -35 & -22 & -57 & -29 & -17 & -46 \\
\hline PPBNGS & 0 & -557 & -557 & 0 & -535 & -535 & $\mathrm{OHPBAT}_{\leftarrow}$ & -17 & -29 & -46 & -22 & -35 & -57 \\
\hline HMBS & -86 & -7 & -93 & -233 & -34 & -267 & AMPMS & -86 & -329 & -415 & -234 & -460 & -694 \\
\hline UPP3S & 0 & -234 & -234 & 0 & -86 & -86 & PMPK & -38 & -34 & -72 & -172 & -139 & -311 \\
\hline UPPDC1 & -449 & -8 & -457 & -325 & -58 & -383 & HMPK 1 & -36 & -358 & -394 & -172 & -589 & -761 \\
\hline CPPPGO & -453 & -241 & -694 & -344 & -144 & -488 & TMPPP & -450 & -8 & -458 & -324 & -74 & -398 \\
\hline PPPGO & -4 & -233 & -237 & -20 & -86 & -106 & $\mathrm{TMPKr}_{\rightarrow}$ & -39 & -35 & -74 & -173 & -140 & -313 \\
\hline FCLT & 0 & -324 & -324 & -2 & -449 & -451 & $\mathrm{TMPKr}_{\leftarrow}$ & -140 & -173 & -313 & -35 & -39 & -74 \\
\hline G1SATi & 0 & 0 & 0 & 0 & 0 & 0 & HETZK & -36 & -358 & -394 & -172 & -589 & -761 \\
\hline UPP3MT & -2 & -325 & -327 & -8 & -455 & -463 & THZPSN & -42 & -587 & -629 & -183 & -706 & -889 \\
\hline IPDDIi & 0 & 0 & 0 & -4 & -1 & -5 & 4HTHRS & -86 & -35 & -121 & -235 & -146 & -381 \\
\hline DMATT & -1 & -7 & -8 & -4 & -72 & -76 & HBZOPT & 0 & -7 & -7 & -1 & -72 & -73 \\
\hline GRTT & 0 & -9 & -9 & -4 & -72 & -76 & OPHHX & -4 & 0 & -4 & -20 & 0 & -20 \\
\hline OCTDPS & 0 & -8 & -8 & -6 & -72 & -78 & CHRPL & 0 & -14 & -14 & -4 & -43 & -47 \\
\hline MEPCT & -451 & -7 & -458 & -330 & -72 & -402 & OPHBDC & -449 & -8 & -457 & -324 & -58 & -382 \\
\hline CDPMEK & -37 & -358 & -395 & -172 & -588 & -760 & OMBZLM & -2 & -325 & -327 & -7 & -455 & -462 \\
\hline MECDPS & 0 & -5 & -5 & 0 & -11 & -11 & OMMBLHX & -4 & 0 & -4 & -20 & 0 & -20 \\
\hline NADDP & -127 & -334 & -461 & -283 & -484 & -767 & ОНРНM & -2 & -325 & -327 & -7 & -455 & -462 \\
\hline DMPPS & -496 & -283 & -779 & -363 & -128 & -491 & DMQMT & -2 & -328 & -330 & -7 & -469 & -476 \\
\hline IPDPS & -496 & -287 & -783 & -363 & -127 & -490 & ОМРHHX & -4 & 0 & -4 & -20 & 0 & -20 \\
\hline DHNAOT & 0 & -344 & -344 & -1 & -584 & -585 & UDCPDPS & 0 & -7 & -7 & -6 & -74 & -80 \\
\hline NPHS & 0 & -21 & -21 & 0 & -23 & -23 & DXYLK & -36 & -360 & -396 & -172 & -589 & -761 \\
\hline SUCBZS & 0 & -233 & -233 & 0 & -86 & -86 & NADK & -78 & -376 & -454 & -222 & -632 & -854 \\
\hline OXGDC2 & -467 & -8 & -475 & -345 & -58 & -403 & BPNT & -87 & -42 & -129 & -233 & -179 & -412 \\
\hline SHCHCS2 & 0 & -15 & -15 & -1 & -44 & -45 & ADSK & -37 & -358 & -395 & -172 & -588 & -760 \\
\hline SUCBZL & -60 & -13 & -73 & -193 & -105 & -298 & SADT2 & -124 & -47 & -171 & -417 & -225 & -642 \\
\hline ICHORSi & 0 & -1 & -1 & -4 & 0 & -4 & $\mathrm{SERAT}_{\rightarrow}$ & -20 & -22 & -42 & -25 & -23 & -48 \\
\hline AMMQT8_2 & -7 & -334 & -341 & -12 & -461 & -473 & $\mathrm{SERAT}_{\leftarrow}$ & -23 & -25 & -48 & -22 & -20 & -42 \\
\hline QULNS & -12 & -593 & -605 & -7 & -681 & -688 & PAPSR & 0 & -324 & -324 & -8 & -461 & -469 \\
\hline ASP03 & -10 & -3 & -13 & -30 & -17 & -47 & $\mathrm{SULR}_{\rightarrow}$ & -131 & -367 & -498 & -252 & -469 & -721 \\
\hline ASPO4 & -13 & -5 & -18 & -26 & -12 & -38 & $\mathrm{SULR}_{\leftarrow}$ & -469 & -252 & -721 & -367 & -131 & -498 \\
\hline ASP05 & -17 & -12 & -29 & -27 & -24 & -51 & CYSS & -1 & -333 & -334 & -2 & -463 & -465 \\
\hline ASP06 & -11 & -1 & -12 & -37 & -7 & -44 & TRPAS1 & -88 & -22 & -110 & -239 & -78 & -317 \\
\hline NNDPR & -450 & -16 & -466 & -335 & -132 & -467 & GCALDD & -127 & -366 & -493 & -283 & -501 & -784 \\
\hline
\end{tabular}




\begin{tabular}{|c|c|c|c|c|c|c|c|c|c|c|c|c|c|}
\hline $\mathrm{MTHFC}_{\rightarrow}$ & -87 & -3 & -90 & -234 & -2 & -236 & $\mathrm{ACCOACr}_{\leftarrow}$ & -735 & -192 & -927 & -395 & -51 & -446 \\
\hline $\mathrm{MTHFC}_{\leftarrow}$ & -2 & -234 & -236 & -3 & -87 & -90 & ACACT1r $\rightarrow$ & -13 & -21 & -34 & -16 & -25 & -41 \\
\hline MTHFD $_{\rightarrow}$ & -46 & -368 & -414 & -21 & -467 & -488 & $\operatorname{ACACT} 1 r_{\leftarrow}$ & -25 & -16 & -41 & -21 & -13 & -34 \\
\hline MTHFD $_{\leftarrow}$ & -467 & -21 & -488 & -368 & -46 & -414 & CDAPPA_EC & -88 & -330 & -418 & -236 & -463 & -699 \\
\hline GLYCL & -51 & -56 & -107 & -59 & -141 & -200 & DASYN_EC $\rightarrow$ & -454 & -10 & -464 & -331 & -74 & -405 \\
\hline MTHFR2 & -498 & -50 & -548 & -365 & -41 & -406 & DASYN_EC $_{\leftarrow}$ & -74 & -331 & -405 & -10 & -454 & -464 \\
\hline FTHFD & -88 & -333 & -421 & -236 & -464 & -700 & CLPNS_EC $\rightarrow$ & -1 & -5 & -6 & -4 & -4 & -8 \\
\hline GLUDC & -483 & -11 & -494 & -352 & -62 & -414 & CLPNS_EC $\leftarrow$ & -4 & -4 & -8 & -5 & -1 & -6 \\
\hline GLUDy $\rightarrow$ & -164 & -394 & -558 & -279 & -517 & -796 & C140SN & -467 & -268 & -735 & -382 & -206 & -588 \\
\hline GLUDy $_{\leftarrow}$ & -517 & -279 & -796 & -394 & -164 & -558 & C120SN & -467 & -267 & -734 & -382 & -205 & -587 \\
\hline GLNS & -105 & -406 & -511 & -207 & -735 & -942 & MACPD & -449 & -9 & -458 & -334 & -59 & -393 \\
\hline GLUSy & -484 & -46 & -530 & -399 & -78 & -477 & KAS14 & -450 & -20 & -470 & -335 & -76 & -411 \\
\hline GLUN & -87 & -35 & -122 & -245 & -68 & -313 & $\mathrm{MCOATA} \rightarrow$ & -18 & -31 & -49 & -8 & -23 & -31 \\
\hline SERD_D & -1 & -21 & -22 & -2 & -77 & -79 & $\mathrm{MCOATA}_{\leftarrow}$ & -23 & -8 & -31 & -31 & -18 & -49 \\
\hline GHMT2 & -12 & -241 & -253 & -13 & -93 & -106 & C160SN & -467 & -266 & -733 & -382 & -206 & -588 \\
\hline GLYATi & -23 & -21 & -44 & -21 & -18 & -39 & KAS16 & -466 & -34 & -500 & -378 & -120 & -498 \\
\hline PGCD & -43 & -362 & -405 & -52 & -496 & -548 & C181SN & -467 & -266 & -733 & -382 & -206 & -588 \\
\hline PSP_L & -86 & -45 & -131 & -233 & -153 & -386 & C141SN & -467 & -266 & -733 & -382 & -206 & -588 \\
\hline PSERT & -34 & -20 & -54 & -28 & -17 & -45 & KAS15 & -462 & -34 & -496 & -350 & -82 & -432 \\
\hline SERD_L & -7 & -21 & -28 & -9 & -77 & -86 & $\mathrm{ACOATA} \rightarrow$ & -30 & -22 & -52 & -23 & -24 & -47 \\
\hline THRD & -46 & -362 & -408 & -56 & -496 & -552 & $\mathrm{ACOATA}_{\leftarrow}$ & -24 & -23 & -47 & -22 & -30 & -52 \\
\hline PDH & -107 & -62 & -169 & -85 & -118 & -203 & C161SN & -467 & -267 & -734 & -382 & -206 & -588 \\
\hline G1PP & -92 & -39 & -131 & -242 & -162 & -404 & FAO2 & -194 & -391 & -585 & -485 & -621 & -1106 \\
\hline ENO $\rightarrow$ & -1 & -253 & -254 & -1 & -89 & -90 & FA01 & -194 & -391 & -585 & -485 & -621 & -1106 \\
\hline $\mathrm{ENO}_{\leftarrow}$ & -89 & -1 & -90 & -253 & -1 & -254 & FAO3 & -188 & -391 & -579 & -484 & -621 & -1105 \\
\hline $\mathrm{FBA} \rightarrow$ & -2 & -16 & -18 & -1 & -21 & -22 & PGPP_EC & -86 & -40 & -126 & -234 & -147 & -381 \\
\hline $\mathrm{FBA}_{\leftarrow}$ & -21 & -1 & -22 & -16 & -2 & -18 & PGSA_EC $\rightarrow$ & -11 & -330 & -341 & -10 & -460 & -470 \\
\hline FBP & -88 & -44 & -132 & -234 & -155 & -389 & $\mathrm{PGSA}_{-} \mathrm{CC}_{\leftarrow}$ & -460 & -10 & -470 & -330 & -11 & -341 \\
\hline $\mathrm{F} 6 \mathrm{PA} \rightarrow$ & -9 & -12 & -21 & -8 & -15 & -23 & PASYN_EC & -14 & -8 & -22 & -10 & -20 & -30 \\
\hline $\mathrm{F}_{6 \mathrm{PA}} \leftarrow$ & -15 & -8 & -23 & -12 & -9 & -21 & PSD_EC & -449 & -9 & -458 & -325 & -59 & -384 \\
\hline $\mathrm{GAPD} \rightarrow$ & -199 & -363 & -562 & -95 & -497 & -592 & PSSA_EC $\rightarrow$ & -9 & -330 & -339 & -12 & -460 & -472 \\
\hline $\mathrm{GAPD}_{\leftarrow}$ & -497 & -95 & -592 & -363 & -199 & -562 & $\mathrm{PSSA}_{-} \mathrm{EC}_{\leftarrow}$ & -460 & -12 & -472 & -330 & -9 & -339 \\
\hline GLCS1 & 0 & -358 & -358 & 0 & -588 & -588 & $\mathrm{AHC}_{\rightarrow}$ & -92 & -7 & -99 & -234 & -7 & -241 \\
\hline GLGC & -492 & -7 & -499 & -505 & -72 & -577 & $\mathrm{AHC}_{\leftarrow}$ & -7 & -234 & -241 & -7 & -92 & -99 \\
\hline GLCP & -146 & -9 & -155 & -36 & -6 & -42 & DHPTDC & 0 & -232 & -232 & 0 & -86 & -86 \\
\hline HEX 1 & -53 & -362 & -415 & -175 & -596 & -771 & RHCCE & 0 & -1 & -1 & 0 & -2 & -2 \\
\hline $\mathrm{PGM}_{\rightarrow}$ & -1 & -2 & -3 & -1 & -2 & -3 & HSST & -2 & -21 & -23 & -6 & -23 & -29 \\
\hline $\mathrm{PGM}_{\leftarrow}$ & -2 & -1 & -3 & -2 & -1 & -3 & SHSL1 & -2 & -336 & -338 & -6 & -470 & -476 \\
\hline PFK & -46 & -359 & -405 & -180 & -590 & -770 & CYSTL & -86 & -22 & -108 & -233 & -79 & -312 \\
\hline $\mathrm{PGI} \rightarrow$ & -8 & -8 & -16 & -4 & -9 & -13 & METS & -2 & -5 & -7 & -1 & -7 & -8 \\
\hline $\mathrm{PGI}_{\leftarrow}$ & -9 & -4 & -13 & -8 & -8 & -16 & METAT & -125 & -50 & -175 & -406 & -220 & -626 \\
\hline $\mathrm{PGK} \rightarrow$ & -39 & -35 & -74 & -174 & -140 & -314 & AHCYSNS & -92 & -5 & -97 & -234 & -6 & -240 \\
\hline $\mathrm{PGK}_{\leftarrow}$ & -140 & -174 & -314 & -35 & -39 & -74 & LGTHL & -3 & 0 & -3 & -2 & 0 & -2 \\
\hline PPS & -166 & -386 & -552 & -419 & -631 & -1050 & GLYOX & -86 & -329 & -415 & -233 & -455 & -688 \\
\hline PYK & -591 & -186 & -777 & -378 & -80 & -458 & MGSA & -9 & -36 & -45 & -7 & -146 & -153 \\
\hline $\mathrm{TPI}_{\rightarrow}$ & -9 & -9 & -18 & -7 & -12 & -19 & UGLYCH & -535 & -18 & -553 & -557 & -96 & -653 \\
\hline $\mathrm{TPI}_{\leftarrow}$ & -12 & -7 & -19 & -9 & -9 & -18 & ALLTN & -87 & -324 & -411 & -235 & -449 & -684 \\
\hline TRSAR & -498 & -50 & -548 & -363 & -43 & -406 & ALLTAH & -86 & -1 & -87 & -233 & -3 & -236 \\
\hline GLXCL & -453 & -9 & -462 & -327 & -60 & -387 & CYNTAH & -450 & -15 & -465 & -329 & -92 & -421 \\
\hline GLYCK & -39 & -360 & -399 & -172 & -590 & -762 & CMPN & -97 & -4 & -101 & -238 & -9 & -247 \\
\hline GLYCLTDy & -470 & -22 & -492 & -370 & -49 & -419 & $\mathrm{ADA}$ & -540 & -11 & -551 & -563 & -39 & -602 \\
\hline GLYCLTDx & -500 & -54 & -554 & -365 & -46 & -411 & DADA & -537 & -8 & -545 & -559 & -36 & -595 \\
\hline PRMICIi & 0 & 0 & 0 & 0 & 0 & 0 & ADNK1 & -42 & -364 & -406 & -178 & -621 & -799 \\
\hline IGPDH & 0 & -233 & -233 & 0 & -86 & -86 & $\mathrm{ADK} 1 \rightarrow$ & -70 & -34 & -104 & -178 & -139 & -317 \\
\hline HISTP & -86 & -36 & -122 & -233 & -146 & -379 & $\mathrm{ADK} 1_{\leftarrow}$ & -139 & -178 & -317 & -34 & -70 & -104 \\
\hline HSTPT & -34 & -20 & -54 & -28 & -17 & -45 & $\mathrm{DADK} \rightarrow$ & -38 & -35 & -73 & -173 & -141 & -314 \\
\hline HISTD & -127 & -364 & -491 & -283 & -499 & -782 & $\mathrm{DADK}_{\leftarrow}$ & -141 & -173 & -314 & -35 & -38 & -73 \\
\hline IG3PS & -1 & -353 & -354 & -12 & -485 & -497 & $\mathrm{ADK} 4 \rightarrow$ & -33 & -34 & -67 & -6 & -139 & -145 \\
\hline ATPPRT & -38 & -7 & -45 & -183 & -72 & -255 & $\mathrm{ADK} 4_{\leftarrow}$ & -139 & -6 & -145 & -34 & -33 & -67 \\
\hline PRATPP & -86 & -331 & -417 & -233 & -521 & -754 & $\mathrm{ADK3} \rightarrow$ & -34 & -38 & -72 & -17 & -146 & -163 \\
\hline PRAMPC & -86 & 0 & -86 & -233 & 0 & -233 & $\mathrm{ADK}_{\leftarrow}$ & -146 & -17 & -163 & -38 & -34 & -72 \\
\hline $\mathrm{PRPPS}_{\rightarrow}$ & -45 & -341 & -386 & -175 & -483 & -658 & AMPN & -119 & -8 & -127 & -239 & -14 & -253 \\
\hline $\mathrm{PRPPS}_{\leftarrow}$ & -483 & -175 & -658 & -341 & -45 & -386 & AP4AH & -85 & -358 & -443 & -233 & -588 & -821 \\
\hline $\mathrm{ACCOACr} \rightarrow$ & -51 & -395 & -446 & -192 & -735 & -927 & GP4GH & -85 & -328 & -413 & -233 & -456 & -689 \\
\hline
\end{tabular}




\begin{tabular}{|c|c|c|c|c|c|c|c|c|c|c|c|c|c|}
\hline AP5AH & -85 & -530 & -615 & -233 & -625 & -858 & TMDK1 & -41 & -359 & -400 & -176 & -591 & -767 \\
\hline $\mathrm{ADPT}$ & -7 & -13 & -20 & -16 & -105 & -121 & TMDS & -4 & -1 & -5 & -5 & -5 & -10 \\
\hline CYTD & -538 & -11 & -549 & -561 & -39 & -600 & $\mathrm{DTMPK}_{\rightarrow}$ & -40 & -35 & -75 & -173 & -141 & -314 \\
\hline DCYTD & -536 & -9 & -545 & -558 & -37 & -595 & DTMPK $_{\leftarrow}$ & -141 & -173 & -314 & -35 & -40 & -75 \\
\hline CYTK2 $\rightarrow$ & -38 & -35 & -73 & -173 & -141 & -314 & URIK2 & -6 & -329 & -335 & -15 & -463 & -478 \\
\hline $\mathrm{CYTK}_{\leftarrow}$ & -141 & -173 & -314 & -35 & -38 & -73 & CYTDK2 & -4 & -333 & -337 & -15 & -467 & -482 \\
\hline CYTK1 $\rightarrow$ & -48 & -36 & -84 & -177 & -141 & -318 & PYNP2r $\rightarrow$ & -151 & -10 & -161 & -40 & -10 & -50 \\
\hline CYTK1 $\leftarrow$ & -141 & -177 & -318 & -36 & -48 & -84 & PYNP2 $r_{\leftarrow}$ & -10 & -40 & -50 & -10 & -151 & -161 \\
\hline $\mathrm{UMPK}_{\rightarrow}$ & -44 & -36 & -80 & -173 & -145 & -318 & UPPRT & -6 & -8 & -14 & -16 & -79 & -95 \\
\hline $\mathrm{UMPK}_{\leftarrow}$ & -145 & -173 & -318 & -36 & -44 & -80 & NTD1 & -88 & -38 & -126 & -235 & -149 & -384 \\
\hline CSND & -536 & -12 & -548 & -558 & -39 & -597 & NTD5 & -89 & -40 & -129 & -234 & -150 & -384 \\
\hline ADNCYC & -37 & -6 & -43 & -172 & -72 & -244 & NTD6 & -87 & -38 & -125 & -234 & -148 & -382 \\
\hline DCTPD & -536 & -8 & -544 & -559 & -36 & -595 & NTD8 & -87 & -37 & -124 & -234 & -149 & -383 \\
\hline $\mathrm{TMDPP} \rightarrow$ & -150 & -5 & -155 & -40 & -5 & -45 & NTD3 & -87 & -37 & -124 & -234 & -147 & -381 \\
\hline $\mathrm{TMDPP}_{\leftarrow}$ & -5 & -40 & -45 & -5 & -150 & -155 & NTD4 & -97 & -40 & -137 & -238 & -149 & -387 \\
\hline DURIPP $_{\rightarrow}$ & -149 & -10 & -159 & -38 & -10 & -48 & NTD7 & -119 & -42 & -161 & -239 & -151 & -390 \\
\hline DURIPP $_{\leftarrow}$ & -10 & -38 & -48 & -10 & -149 & -159 & NTD9 & -91 & -38 & -129 & -235 & -149 & -384 \\
\hline NTPTP1 & -87 & 0 & -87 & -235 & -4 & -239 & NTD11 & -89 & -40 & -129 & -236 & -151 & -387 \\
\hline NTPTP2 & -87 & -1 & -88 & -244 & -4 & -248 & NTD10 & -87 & -38 & -125 & -234 & -149 & -383 \\
\hline DUTPDP & -88 & -333 & -421 & -234 & -523 & -757 & NTD2 & -93 & -40 & -133 & -234 & -151 & -385 \\
\hline GK1 $\rightarrow$ & -42 & -38 & -80 & -174 & -146 & -320 & PUNP6 $\rightarrow$ & -148 & -9 & -157 & -37 & -9 & -46 \\
\hline $\mathrm{GK} 1_{\leftarrow}$ & -146 & -174 & -320 & -38 & -42 & -80 & PUNP6 $_{\leftarrow}$ & -9 & -37 & -46 & -9 & -148 & -157 \\
\hline DGK1 $\rightarrow$ & -38 & -35 & -73 & -173 & -141 & -314 & PUNP5 $\rightarrow$ & -151 & -9 & -160 & -40 & -9 & -49 \\
\hline $\mathrm{DGK} 1_{\leftarrow}$ & -141 & -173 & -314 & -35 & -38 & -73 & PUNP5 $_{\leftarrow}$ & -9 & -40 & -49 & -9 & -151 & -160 \\
\hline XPPT & -5 & -8 & -13 & -15 & -73 & -88 & PUNP2 $\rightarrow$ & -148 & -10 & -158 & -38 & -11 & -49 \\
\hline HXPRT & -5 & -10 & -15 & -15 & -75 & -90 & PUNP2 $\leftarrow$ & -11 & -38 & -49 & -10 & -148 & -158 \\
\hline GUAPRT & -5 & -9 & -14 & -17 & -77 & -94 & PUNP4 $\rightarrow$ & -149 & -11 & -160 & -37 & -9 & -46 \\
\hline INSK & -42 & -361 & -403 & -176 & -591 & -767 & $\mathrm{PUNP}_{\leftarrow}$ & -9 & -37 & -46 & -11 & -149 & -160 \\
\hline GSNK & -40 & -360 & -400 & -174 & -593 & -767 & PUNP1 $\rightarrow$ & -151 & -10 & -161 & -42 & -11 & -53 \\
\hline NTPP3 & -87 & -332 & -419 & -235 & -522 & -757 & PUNP1 $\leftarrow$ & -11 & -42 & -53 & -10 & -151 & -161 \\
\hline NTPP4 & -88 & -336 & -424 & -239 & -532 & -771 & PUNP3 $\rightarrow$ & -149 & -11 & -160 & -38 & -9 & -47 \\
\hline NTPP5 & -87 & -332 & -419 & -234 & -522 & -756 & $\mathrm{PUNP}_{\leftarrow} \leftarrow$ & -9 & -38 & -47 & -11 & -149 & -160 \\
\hline NTPP6 & -123 & -337 & -460 & -405 & -554 & -959 & PUNP7 $\rightarrow$ & -149 & -9 & -158 & -38 & -9 & -47 \\
\hline NTPP7 & -86 & -332 & -418 & -235 & -524 & -759 & PUNP7 $\leftarrow$ & -9 & -38 & -47 & -9 & -149 & -158 \\
\hline NTPP8 & -87 & -332 & -419 & -239 & -528 & -767 & GUAD & -539 & -11 & -550 & -563 & -38 & -601 \\
\hline NTPP1 & -87 & -332 & -419 & -235 & -522 & -757 & $\mathrm{ADD}$ & -541 & -11 & -552 & -562 & -38 & -600 \\
\hline NTPP2 & -87 & -333 & -420 & -244 & -526 & -770 & L-LACD2 & -6 & -17 & -23 & -17 & -57 & -74 \\
\hline NDPK1 $\rightarrow$ & -44 & -45 & -89 & -176 & -140 & -316 & L-LACD3 & -9 & -19 & -28 & -13 & -52 & -65 \\
\hline $\mathrm{NDPK} 1_{\leftarrow}$ & -140 & -176 & -316 & -45 & -44 & -89 & ATPS4r $\rightarrow$ & -734 & -729 & -1463 & -394 & -572 & -966 \\
\hline $\mathrm{NDPK} 2 \rightarrow$ & -43 & -40 & -83 & -174 & -140 & -314 & $\operatorname{ATPS} 4 r_{\leftarrow}$ & -572 & -394 & -966 & -729 & -734 & -1463 \\
\hline NDPK $2_{\leftarrow}$ & -140 & -174 & -314 & -40 & -43 & -83 & $\mathrm{CRNBTCT}_{\rightarrow}$ & -2 & -3 & -5 & -2 & -3 & -5 \\
\hline NDPK3 $\rightarrow$ & -39 & -40 & -79 & -174 & -141 & -315 & CRNBTCT $_{\leftarrow}$ & -3 & -2 & -5 & -3 & -2 & -5 \\
\hline NDPK3 $\leftarrow$ & -141 & -174 & -315 & -40 & -39 & -79 & $\mathrm{CRNCBCT}_{\rightarrow}$ & -3 & -2 & -5 & -3 & -2 & -5 \\
\hline NDPK5 $\rightarrow$ & -39 & -36 & -75 & -173 & -140 & -313 & $\mathrm{CRNCBCT}_{\leftarrow}$ & -2 & -3 & -5 & -2 & -3 & -5 \\
\hline $\mathrm{NDPK}_{\leftarrow} \leftarrow$ & -140 & -173 & -313 & -36 & -39 & -75 & $\mathrm{CRNCDH}_{\rightarrow}$ & -2 & -234 & -236 & -2 & -87 & -89 \\
\hline NDPK6 $\rightarrow$ & -39 & -35 & -74 & -173 & -141 & -314 & $\mathrm{CRNCDH}_{\leftarrow}$ & -87 & -2 & -89 & -234 & -2 & -236 \\
\hline $\mathrm{NDPK}_{\leftarrow}$ & -141 & -173 & -314 & -35 & -39 & -74 & CYTBD & -467 & -570 & -1037 & -347 & -538 & -885 \\
\hline NDPK7 $\rightarrow$ & -39 & -36 & -75 & -173 & -140 & -313 & СҮТВОЗ & -467 & -570 & -1037 & -347 & -538 & -885 \\
\hline NDPK7 $\leftarrow$ & -140 & -173 & -313 & -36 & -39 & -75 & LDH_D2 & -6 & -17 & -23 & -16 & -57 & -73 \\
\hline NDPK8 $\rightarrow$ & -39 & -35 & -74 & -173 & -140 & -313 & DMSOR1 & -8 & -241 & -249 & -8 & -95 & -103 \\
\hline $\mathrm{NDPK}_{\leftarrow}$ & -140 & -173 & -313 & -35 & -39 & -74 & DMSOR2 & -3 & -237 & -240 & -7 & -94 & -101 \\
\hline $\mathrm{NDPK} 4 \rightarrow$ & -39 & -36 & -75 & -173 & -139 & -312 & TMAOR1 & -457 & -241 & -698 & -332 & -95 & -427 \\
\hline $\mathrm{NDPK}_{\leftarrow}$ & -139 & -173 & -312 & -36 & -39 & -75 & TMAOR2 & -452 & -237 & -689 & -331 & -94 & -425 \\
\hline RNDR1 & -139 & -234 & -373 & -42 & -96 & -138 & FDH2 & -462 & -335 & -797 & -342 & -521 & -863 \\
\hline RNDR2 & -7 & -234 & -241 & -12 & -96 & -108 & FDH3 & -465 & -337 & -802 & -338 & -516 & -854 \\
\hline RNDR4 & -6 & -234 & -240 & -10 & -96 & -106 & GLCDe & -105 & -330 & -435 & -249 & -468 & -717 \\
\hline RNDR3 & -2 & -234 & -236 & -10 & -96 & -106 & G3PD7 & -14 & -11 & -25 & -12 & -13 & -25 \\
\hline RNTR1 & -37 & -234 & -271 & -180 & -95 & -275 & G3PD6 & -15 & -12 & -27 & -16 & -18 & -34 \\
\hline RNTR2 & -1 & -235 & -236 & -19 & -95 & -114 & G3PD5 & -12 & -10 & -22 & -20 & -23 & -43 \\
\hline RNTR3 & -2 & -235 & -237 & -14 & -95 & -109 & HYD3 & -454 & -328 & -782 & -331 & -453 & -784 \\
\hline RNTR4 & -1 & -234 & -235 & -14 & -96 & -110 & HYD1 & -452 & -327 & -779 & -339 & -463 & -802 \\
\hline URIDK2r $\rightarrow$ & -39 & -35 & -74 & -174 & -141 & -315 & HYD2 & -455 & -329 & -784 & -335 & -458 & -793 \\
\hline URIDK2r $r_{\leftarrow}$ & -141 & -174 & -315 & -35 & -39 & -74 & N03R1 & -463 & -573 & -1036 & -329 & -542 & -871 \\
\hline DURIK1 & -40 & -360 & -400 & -174 & -590 & -764 & N03R2 & -458 & -569 & -1027 & -331 & -545 & -876 \\
\hline
\end{tabular}




\begin{tabular}{|c|c|c|c|c|c|c|c|c|c|c|c|c|c|}
\hline NADH5 & -499 & -53 & -552 & -375 & -55 & -430 & $\mathrm{ORPT}_{\leftarrow}$ & -3 & -8 & -11 & -11 & -72 & -83 \\
\hline NADH9 & -501 & -54 & -555 & -367 & -45 & -412 & OMPDC & -449 & -9 & -458 & -325 & -65 & -390 \\
\hline NADH10 & -502 & -55 & -557 & -371 & -50 & -421 & CTPS2 & -125 & -428 & -553 & -423 & -770 & -1193 \\
\hline NTRIR2x & -500 & -290 & -790 & -365 & -161 & -526 & CBMK & -129 & -359 & -488 & -187 & -590 & -777 \\
\hline NADH6 & -499 & -377 & -876 & -375 & -504 & -879 & AMANAPE & -1 & 0 & -1 & 0 & -1 & -1 \\
\hline NADH7 & -502 & -379 & -881 & -371 & -499 & -870 & AMANK & -37 & -358 & -395 & -173 & -589 & -762 \\
\hline NADH8 & -501 & -378 & -879 & -367 & -494 & -861 & ALLTNt $2 r \rightarrow$ & -450 & -326 & -776 & -326 & -450 & -776 \\
\hline THD2 & -540 & -417 & -957 & -380 & -507 & -887 & ALLTNt $2 r_{\leftarrow}$ & -450 & -326 & -776 & -326 & -450 & -776 \\
\hline POX & -132 & -14 & -146 & -260 & -84 & -344 & ARGORNt $7 \rightarrow$ & -9 & -12 & -21 & -12 & -9 & -21 \\
\hline SUCD4 $\rightarrow$ & -10 & -10 & -20 & -13 & -15 & -28 & ARGORNt $7_{\leftarrow}$ & -9 & -12 & -21 & -12 & -9 & -21 \\
\hline SUCD4 $\leftarrow$ & -15 & -13 & -28 & -10 & -10 & -20 & ACACt $2 \rightarrow$ & -450 & -326 & -776 & -326 & -450 & -776 \\
\hline EST_NADTRHD & -58 & -56 & -114 & -93 & -91 & -184 & $\mathrm{ACACt} 2_{\leftarrow}$ & -450 & -326 & -776 & -326 & -450 & -776 \\
\hline TMAOR1e & -457 & -241 & -698 & -332 & -95 & -427 & BUTt $2 r \rightarrow$ & -450 & -326 & -776 & -326 & -450 & -776 \\
\hline TMAOR2e & -452 & -237 & -689 & -331 & -94 & -425 & BUTt $2 r_{\leftarrow}$ & -450 & -326 & -776 & -326 & -450 & -776 \\
\hline DMSOR1e & -8 & -241 & -249 & -8 & -95 & -103 & CYNTt2 & -449 & -325 & -774 & -325 & -449 & -774 \\
\hline DMSOR2e & -3 & -237 & -240 & -7 & -94 & -101 & GALCTt2r $\rightarrow$ & -450 & -326 & -776 & -326 & -450 & -776 \\
\hline TRDR & -474 & -26 & -500 & -367 & -44 & -411 & GALCTt $2 r_{\leftarrow}$ & -450 & -326 & -776 & -326 & -450 & -776 \\
\hline PGL & -86 & -325 & -411 & -234 & -450 & -684 & GLCRt $2 r \rightarrow$ & -450 & -326 & -776 & -326 & -450 & -776 \\
\hline EDA & -1 & -23 & -24 & 0 & -55 & -55 & GLCRt $2 r_{\leftarrow}$ & -450 & -326 & -776 & -326 & -450 & -776 \\
\hline EDD & -1 & -233 & -234 & -1 & -87 & -88 & PPPNt2r $\rightarrow$ & -450 & -326 & -776 & -326 & -450 & -776 \\
\hline GND & -45 & -54 & -99 & -19 & -78 & -97 & PPPNt2r $\leftarrow$ & -450 & -326 & -776 & -326 & -450 & -776 \\
\hline $\mathrm{RPE}_{\rightarrow}$ & -3 & -3 & -6 & -3 & -4 & -7 & HPPPNt $2 r \rightarrow$ & -450 & -326 & -776 & -326 & -450 & -776 \\
\hline $\mathrm{RPE}_{\leftarrow}$ & -4 & -3 & -7 & -3 & -3 & -6 & HPPPNt $2 r_{\leftarrow}$ & -450 & -326 & -776 & -326 & -450 & -776 \\
\hline $\mathrm{RPI}_{\rightarrow}$ & -8 & -3 & -11 & -3 & -3 & -6 & HCINNMt $2 r \rightarrow$ & -450 & -326 & -776 & -326 & -450 & -776 \\
\hline $\mathrm{RPI}_{\leftarrow}$ & -3 & -3 & -6 & -3 & -8 & -11 & HCINNMt $2 r_{\leftarrow}$ & -450 & -326 & -776 & -326 & -450 & -776 \\
\hline TALA $\rightarrow$ & -13 & -11 & -24 & -11 & -11 & -22 & GLUABUTt $7 \rightarrow$ & -38 & -31 & -69 & -31 & -38 & -69 \\
\hline TALA $_{\leftarrow}$ & -11 & -11 & -22 & -11 & -13 & -24 & GLUABUTt $7 \leftarrow$ & -38 & -31 & -69 & -31 & -38 & -69 \\
\hline TKT1 $\rightarrow$ & -12 & -11 & -23 & -6 & -13 & -19 & ALAt2r $\rightarrow$ & -456 & -334 & -790 & -334 & -456 & -790 \\
\hline TKT $1_{\leftarrow}$ & -13 & -6 & -19 & -11 & -12 & -23 & ALAt $2 r_{\leftarrow}$ & -456 & -334 & -790 & -334 & -456 & -790 \\
\hline TKT2 $\rightarrow$ & -6 & -17 & -23 & -6 & -21 & -27 & URAt $2 r \rightarrow$ & -454 & -329 & -783 & -329 & -454 & -783 \\
\hline TKT $2_{\leftarrow}$ & -21 & -6 & -27 & -17 & -6 & -23 & URAt $2 r_{\leftarrow}$ & -454 & -329 & -783 & -329 & -454 & -783 \\
\hline G6PDH2r $\rightarrow$ & -52 & -368 & -420 & -22 & -466 & -488 & GLYBt $2 r \rightarrow$ & -453 & -326 & -779 & -326 & -453 & -779 \\
\hline $\mathrm{G} 6 \mathrm{PDH} 2 r_{\leftarrow}$ & -466 & -22 & -488 & -368 & -52 & -420 & GLYBt $2 r_{\leftarrow}$ & -453 & -326 & -779 & -326 & -453 & -779 \\
\hline GMPS2 & -125 & -367 & -492 & -418 & -593 & -1011 & CHLabc & -126 & -396 & -522 & -407 & -737 & -1144 \\
\hline IMPD & -130 & -363 & -493 & -286 & -497 & -783 & GLYBabc & -127 & -396 & -523 & -407 & -738 & -1145 \\
\hline GMPR & -471 & -28 & -499 & -369 & -81 & -450 & TARTRt $7 \rightarrow$ & -22 & -14 & -36 & -14 & -22 & -36 \\
\hline ADSS & -11 & -364 & -375 & -31 & -603 & -634 & TARTRt $7_{\leftarrow}$ & -22 & -14 & -36 & -14 & -22 & -36 \\
\hline $\operatorname{ADSL} 2 r \rightarrow$ & -1 & -11 & -12 & -1 & -12 & -13 & SUCCabc & -144 & -406 & -550 & -417 & -755 & -1172 \\
\hline $\operatorname{ADSL} 2 r_{\leftarrow}$ & -12 & -1 & -13 & -11 & -1 & -12 & GUAt2 & -453 & -330 & -783 & -330 & -453 & -783 \\
\hline $\operatorname{ADSL} 1 r \rightarrow$ & -1 & -16 & -17 & 0 & -43 & -43 & XANt 2 & -453 & -328 & -781 & -328 & -453 & -781 \\
\hline $\operatorname{ADSL} 1 r_{\leftarrow}$ & -43 & 0 & -43 & -16 & -1 & -17 & $\mathrm{ACKr} \rightarrow$ & -49 & -35 & -84 & -175 & -140 & -315 \\
\hline PRASCS $_{\rightarrow}$ & -45 & -395 & -440 & -190 & -735 & -925 & $\mathrm{ACKr}_{\leftarrow}$ & -140 & -175 & -315 & -35 & -49 & -84 \\
\hline PRASCS $_{\leftarrow}$ & -735 & -190 & -925 & -395 & -45 & -440 & ACS & -72 & -29 & -101 & -196 & -118 & -314 \\
\hline PRAGSr $\rightarrow$ & -43 & -396 & -439 & -177 & -735 & -912 & $\mathrm{ADHEr}_{\rightarrow} \rightarrow$ & -509 & -73 & -582 & -378 & -66 & -444 \\
\hline PRAGSr $_{\leftarrow}$ & -735 & -177 & -912 & -396 & -43 & -439 & $\mathrm{ADHEr}_{\leftarrow}$ & -66 & -378 & -444 & -73 & -509 & -582 \\
\hline AIRC3 $\rightarrow$ & -1 & 0 & -1 & -1 & -1 & -2 & LDH_D $\rightarrow$ & -44 & -376 & -420 & -53 & -539 & -592 \\
\hline $\mathrm{AIRC}_{\leftarrow} \leftarrow$ & -1 & -1 & -2 & 0 & -1 & -1 & $\mathrm{LDH}_{-} \mathrm{D}_{\leftarrow}$ & -539 & -53 & -592 & -376 & -44 & -420 \\
\hline GLUPRT & -88 & -35 & -123 & -256 & -107 & -363 & FHL & -459 & -10 & -469 & -329 & -58 & -387 \\
\hline $\mathrm{AICART}_{\rightarrow}$ & -4 & -5 & -9 & -4 & -6 & -10 & $\mathrm{PTAr} \rightarrow$ & -159 & -22 & -181 & -52 & -24 & -76 \\
\hline AICART $_{\leftarrow}$ & -6 & -4 & -10 & -5 & -4 & -9 & $\mathrm{PTAr}_{\leftarrow}$ & -24 & -52 & -76 & -22 & -159 & -181 \\
\hline IMPC $_{\rightarrow}$ & -89 & -1 & -90 & -236 & -1 & -237 & PFL & -66 & -21 & -87 & -35 & -23 & -58 \\
\hline $\operatorname{IMPC}_{\leftarrow}$ & -1 & -236 & -237 & -1 & -89 & -90 & $\mathrm{SDPTA}_{\rightarrow}$ & -17 & -28 & -45 & -21 & -35 & -56 \\
\hline AIRC2 & -38 & -394 & -432 & -177 & -735 & -912 & $\mathrm{SDPTA}_{\leftarrow}$ & -35 & -21 & -56 & -28 & -17 & -45 \\
\hline PRFGS & -125 & -422 & -547 & -418 & -768 & -1186 & $\mathrm{ASAD}_{\rightarrow}$ & -191 & -368 & -559 & -56 & -467 & -523 \\
\hline PRAIS & -37 & -395 & -432 & -172 & -734 & -906 & $\mathrm{ASAD}_{\leftarrow}$ & -467 & -56 & -523 & -368 & -191 & -559 \\
\hline $\mathrm{GARFT}_{\rightarrow}$ & -3 & -329 & -332 & -5 & -455 & -460 & DHDPS & -44 & -557 & -601 & -16 & -535 & -551 \\
\hline $\mathrm{GARFT}_{\leftarrow}$ & -455 & -5 & -460 & -329 & -3 & -332 & DHDPRy & -466 & -18 & -484 & -367 & -44 & -411 \\
\hline GART & -48 & -395 & -443 & -179 & -735 & -914 & THDPS & -88 & -21 & -109 & -237 & -24 & -261 \\
\hline ASPCT & -9 & -360 & -369 & -18 & -596 & -614 & SDPDS & -86 & -12 & -98 & -234 & -22 & -256 \\
\hline DHORTS $_{\rightarrow}$ & -86 & -324 & -410 & -235 & -450 & -685 & $\mathrm{DAPE}_{\rightarrow}$ & -1 & -3 & -4 & 0 & -1 & -1 \\
\hline DHORTS $_{\leftarrow}$ & -450 & -235 & -685 & -324 & -86 & -410 & $\mathrm{DAPE}_{\leftarrow}$ & -1 & 0 & -1 & -3 & -1 & -4 \\
\hline DHORD2 & -3 & -3 & -6 & -15 & -16 & -31 & LYSDC & -453 & -8 & -461 & -328 & -59 & -387 \\
\hline DHORD5 & -6 & -5 & -11 & -11 & -11 & -22 & THRAr $\rightarrow$ & -5 & -9 & -14 & -6 & -10 & -16 \\
\hline $\mathrm{ORPT}_{\rightarrow}$ & -72 & -11 & -83 & -8 & -3 & -11 & THRAr $_{\leftarrow}$ & -10 & -6 & -16 & -9 & -5 & -14 \\
\hline
\end{tabular}




\begin{tabular}{|c|c|c|c|c|c|c|c|c|c|c|c|c|c|}
\hline DAPDC & -450 & -12 & -462 & -327 & -62 & -389 & DSERt $2 r \rightarrow$ & -450 & -326 & -776 & -326 & -450 & -776 \\
\hline HSDy $\rightarrow$ & -44 & -369 & -413 & -20 & -467 & -487 & DSERt $2 r_{\leftarrow}$ & -450 & -326 & -776 & -326 & -450 & -776 \\
\hline $\mathrm{HSDy}_{\leftarrow}$ & -467 & -20 & -487 & -369 & -44 & -413 & GLYt $2 r_{\rightarrow}$ & -454 & -329 & -783 & -329 & -454 & -783 \\
\hline $\mathrm{ASPK}_{\rightarrow}$ & -44 & -35 & -79 & -189 & -140 & -329 & GLYt $2 r_{\leftarrow}$ & -454 & -329 & -783 & -329 & -454 & -783 \\
\hline $\mathrm{ASPK}_{\leftarrow}$ & -140 & -189 & -329 & -35 & -44 & -79 & SULabc & -123 & -395 & -518 & -406 & -734 & -1140 \\
\hline HSK & -37 & -358 & -395 & -174 & -588 & -762 & ASPt2_2 & -456 & -341 & -797 & -341 & -456 & -797 \\
\hline THRS & -86 & -42 & -128 & -233 & -151 & -384 & FUMt2_2 & -459 & -334 & -793 & -334 & -459 & -793 \\
\hline $12 \mathrm{PPDt} \rightarrow$ & -2 & -2 & -4 & -2 & -2 & -4 & MALt2_2 & -453 & -331 & -784 & -331 & -453 & -784 \\
\hline $12 \mathrm{PPDt}_{\leftarrow}$ & -2 & -2 & -4 & -2 & -2 & -4 & SUCCt2_2 & -470 & -336 & -806 & -336 & -470 & -806 \\
\hline NMNt7 & -88 & -327 & -415 & -237 & -458 & -695 & ASPt2_3 & -456 & -341 & -797 & -341 & -456 & -797 \\
\hline ACALDt $_{\rightarrow}$ & -5 & -4 & -9 & -4 & -5 & -9 & MALt2_3 & -453 & -331 & -784 & -331 & -453 & -784 \\
\hline ACALDt $_{\leftarrow}$ & -5 & -4 & -9 & -4 & -5 & -9 & SUCCt2_3 & -470 & -336 & -806 & -336 & -470 & -806 \\
\hline GUAt $\rightarrow$ & -4 & -6 & -10 & -6 & -4 & -10 & SUCCt $2 b$ & -470 & -336 & -806 & -336 & -470 & -806 \\
\hline GUAt $_{\leftarrow}$ & -4 & -6 & -10 & -6 & -4 & -10 & FUMt2_3 & -459 & -334 & -793 & -334 & -459 & -793 \\
\hline $\mathrm{HYXNt}_{\rightarrow}$ & -4 & -4 & -8 & -4 & -4 & -8 & SUCFUMt $_{\rightarrow}$ & -31 & -22 & -53 & -22 & -31 & -53 \\
\hline HYXNt $_{\leftarrow}$ & -4 & -4 & -8 & -4 & -4 & -8 & SUCFUMt $_{\leftarrow}$ & -31 & -22 & -53 & -22 & -31 & -53 \\
\hline $\mathrm{XANt}_{\rightarrow}$ & -4 & -4 & -8 & -4 & -4 & -8 & GALCTNt $2 r \rightarrow$ & -450 & -326 & -776 & -326 & -450 & -776 \\
\hline XANt $_{\leftarrow}$ & -4 & -4 & -8 & -4 & -4 & -8 & GALCTNt $2 r_{\leftarrow}$ & -450 & -326 & -776 & -326 & -450 & -776 \\
\hline NACUP & -2 & -1 & -3 & -1 & -2 & -3 & GALURt2r $\rightarrow$ & -451 & -326 & -777 & -326 & -451 & -777 \\
\hline ASNabc & -127 & -397 & -524 & -408 & -738 & -1146 & GALURt $2 r_{\leftarrow}$ & -451 & -326 & -777 & -326 & -451 & -777 \\
\hline ASNt2r $\rightarrow$ & -453 & -327 & -780 & -327 & -453 & -780 & GLCURt $2 r \rightarrow$ & -451 & -326 & -777 & -326 & -451 & -777 \\
\hline ASNt $2 r_{\leftarrow}$ & -453 & -327 & -780 & -327 & -453 & -780 & GLCURt $2 r_{\leftarrow}$ & -451 & -326 & -777 & -326 & -451 & -777 \\
\hline DAPabc & -124 & -397 & -521 & -408 & -735 & -1143 & OCDCAt2 & -449 & -325 & -774 & -325 & -449 & -774 \\
\hline CYSabc & -125 & -400 & -525 & -411 & -736 & -1147 & HDCAt2 & -455 & -326 & -781 & -326 & -455 & -781 \\
\hline $\mathrm{ACt} 2 \mathrm{r} \rightarrow$ & -461 & -327 & -788 & -327 & -461 & -788 & TTDCAt2 & -455 & -326 & -781 & -326 & -455 & -781 \\
\hline $\mathrm{ACt} 2 \mathrm{r}_{\leftarrow}$ & -461 & -327 & -788 & -327 & -461 & -788 & FE2abc & -123 & -396 & -519 & -407 & -734 & -1141 \\
\hline ETOHt2r $\rightarrow$ & -451 & -326 & -777 & -326 & -451 & -777 & FORt $\rightarrow$ & -10 & -5 & -15 & -5 & -10 & -15 \\
\hline ETOHt2r $r_{\leftarrow}$ & -451 & -326 & -777 & -326 & -451 & -777 & FORt $_{\leftarrow}$ & -10 & -5 & -15 & -5 & -10 & -15 \\
\hline PYRt2r $\rightarrow$ & -492 & -338 & -830 & -338 & -492 & -830 & FUCt $\rightarrow$ & -451 & -326 & -777 & -326 & -451 & -777 \\
\hline PYRt $2 r_{\leftarrow}$ & -492 & -338 & -830 & -338 & -492 & -830 & FUCt $_{\leftarrow}$ & -451 & -326 & -777 & -326 & -451 & -777 \\
\hline $02 t \rightarrow$ & -4 & -20 & -24 & -20 & -4 & -24 & ABUTt2 & -453 & -327 & -780 & -327 & -453 & -780 \\
\hline $02 t_{\leftarrow}$ & -4 & -20 & -24 & -20 & -4 & -24 & GALt2 & -453 & -326 & -779 & -326 & -453 & -779 \\
\hline $\mathrm{CO} 2 \mathrm{t}_{\rightarrow}$ & -58 & -8 & -66 & -8 & -58 & -66 & GLCt2 & -465 & -327 & -792 & -327 & -465 & -792 \\
\hline $\mathrm{CO} 2 \mathrm{t}_{\leftarrow}$ & -58 & -8 & -66 & -8 & -58 & -66 & GALTpts & -2 & -14 & -16 & -20 & -44 & -64 \\
\hline $\mathrm{H} 2 \mathrm{Ot} \rightarrow$ & -86 & -233 & -319 & -233 & -86 & -319 & GLNabc & -124 & -406 & -530 & -417 & -735 & -1152 \\
\hline $\mathrm{H} 20 \mathrm{t}_{\leftarrow}$ & -86 & -233 & -319 & -233 & -86 & -319 & GLYCt $_{\rightarrow}$ & -4 & -5 & -9 & -5 & -4 & -9 \\
\hline DHAt $_{\rightarrow}$ & -3 & -3 & -6 & -3 & -3 & -6 & GLYCt $_{\leftarrow}$ & -4 & -5 & -9 & -5 & -4 & -9 \\
\hline DHAt $_{\leftarrow}$ & -3 & -3 & -6 & -3 & -3 & -6 & GLYALDt $_{\rightarrow}$ & -2 & -2 & -4 & -2 & -2 & -4 \\
\hline NH3t $\rightarrow$ & -34 & -7 & -41 & -7 & -34 & -41 & GLYALDt $_{\leftarrow}$ & -2 & -2 & -4 & -2 & -2 & -4 \\
\hline $\mathrm{NH} \mathrm{t}_{\leftarrow}$ & -34 & -7 & -41 & -7 & -34 & -41 & UREAt $_{\rightarrow}$ & -3 & -1 & -4 & -1 & -3 & -4 \\
\hline ARBt $2 r \rightarrow$ & -452 & -327 & -779 & -327 & -452 & -779 & UREAt $_{\leftarrow}$ & -3 & -1 & -4 & -1 & -3 & -4 \\
\hline ARBt $2 r_{\leftarrow}$ & -452 & -327 & -779 & -327 & -452 & -779 & GLYC3Pt6 & -155 & -43 & -198 & -43 & -155 & -198 \\
\hline $\mathrm{ARBabc}$ & -126 & -397 & -523 & -408 & -737 & -1145 & ASPabc & -130 & -411 & -541 & -422 & -741 & -1163 \\
\hline HISt $2 r \rightarrow$ & -452 & -326 & -778 & -326 & -452 & -778 & GLUabc & -157 & -422 & -579 & -433 & -768 & -1201 \\
\hline HISt $2 r_{\leftarrow}$ & -452 & -326 & -778 & -326 & -452 & -778 & ASPt2 & -456 & -341 & -797 & -341 & -456 & -797 \\
\hline PHEt2r $\rightarrow$ & -451 & -326 & -777 & -326 & -451 & -777 & GLUt $2 r \rightarrow$ & -483 & -352 & -835 & -352 & -483 & -835 \\
\hline PHEt $2 r_{\leftarrow}$ & -451 & -326 & -777 & -326 & -451 & -777 & GLUt $2 r_{\leftarrow}$ & -483 & -352 & -835 & -352 & -483 & -835 \\
\hline LEUt2r $\rightarrow$ & -452 & -326 & -778 & -326 & -452 & -778 & GLUt4 & -42 & -36 & -78 & -36 & -42 & -78 \\
\hline LEUt $2 r_{\leftarrow}$ & -452 & -326 & -778 & -326 & -452 & -778 & ORNabc & -129 & -401 & -530 & -412 & -740 & -1152 \\
\hline VALt $2 r \rightarrow$ & -453 & -327 & -780 & -327 & -453 & -780 & ARGabc & -126 & -399 & -525 & -410 & -737 & -1147 \\
\hline VALt $2 r_{\leftarrow}$ & -453 & -327 & -780 & -327 & -453 & -780 & HISabc & -126 & -396 & -522 & -407 & -737 & -1144 \\
\hline ILEt2r $\rightarrow$ & -452 & -327 & -779 & -327 & -452 & -779 & LYSabc & -127 & -398 & -525 & -409 & -738 & -1147 \\
\hline ILEt $2 r_{\leftarrow}$ & -452 & -327 & -779 & -327 & -452 & -779 & IDONt $2 r \rightarrow$ & -454 & -326 & -780 & -326 & -454 & -780 \\
\hline CBL1abc & -124 & -395 & -519 & -406 & -735 & -1141 & IDONt $2 r_{\leftarrow}$ & -454 & -326 & -780 & -326 & -454 & -780 \\
\hline CADVt & -454 & -328 & -782 & -328 & -454 & -782 & GLCNt2r $\rightarrow$ & -454 & -327 & -781 & -327 & -454 & -781 \\
\hline CRNt7 & -3 & -3 & -6 & -3 & -3 & -6 & GLCNt $2 r_{\leftarrow}$ & -454 & -327 & -781 & -327 & -454 & -781 \\
\hline NAt3_1 $\rightarrow$ & -457 & -332 & -789 & -332 & -457 & -789 & DDGLCNt $2 r \rightarrow$ & -452 & -326 & -778 & -326 & -452 & -778 \\
\hline NAt3_1 $\leftarrow$ & -457 & -332 & -789 & -332 & -457 & -789 & DDGLCNt $2 r_{\leftarrow}$ & -452 & -326 & -778 & -326 & -452 & -778 \\
\hline CITt7 & -23 & -14 & -37 & -14 & -23 & -37 & $\mathrm{Kabc}$ & -125 & -396 & -521 & -407 & -736 & -1143 \\
\hline CSNt2 & -450 & -325 & -775 & -325 & -450 & -775 & $\operatorname{AKGt} 2 r_{\rightarrow}$ & -466 & -344 & -810 & -344 & -466 & -810 \\
\hline MALTpts & -4 & -13 & -17 & -25 & -43 & -68 & $\operatorname{AKGt} 2 r_{\leftarrow}$ & -466 & -344 & -810 & -344 & -466 & -810 \\
\hline ACGApts & -2 & -14 & -16 & -20 & -44 & -64 & LCTSt $_{\rightarrow}$ & -450 & -326 & -776 & -326 & -450 & -776 \\
\hline DALAt $2 r \rightarrow$ & -453 & -329 & -782 & -329 & -453 & -782 & LCTSt $_{\leftarrow}$ & -450 & -326 & -776 & -326 & -450 & -776 \\
\hline DALAt $2 r_{\leftarrow}$ & -453 & -329 & -782 & -329 & -453 & -782 & ILEabc & -126 & -397 & -523 & -408 & -737 & -1145 \\
\hline
\end{tabular}




\begin{tabular}{|c|c|c|c|c|c|c|c|c|c|c|c|c|c|}
\hline THRabc & -128 & -400 & -528 & -411 & -739 & -1150 & RMNt & -450 & -325 & -775 & -325 & -450 & -775 \\
\hline ALAabc & -130 & -404 & -534 & -415 & -741 & -1156 & TSULabc & -123 & -395 & -518 & -406 & -734 & -1140 \\
\hline VALabc & -127 & -397 & -524 & -408 & -738 & -1146 & SERt $2 r_{\rightarrow}$ & -456 & -333 & -789 & -333 & -456 & -789 \\
\hline LEUabc & -126 & -396 & -522 & -407 & -737 & -1144 & SERt $2 r_{\leftarrow}$ & -456 & -333 & -789 & -333 & -456 & -789 \\
\hline D-LACt $2 \rightarrow$ & -452 & -327 & -779 & -327 & -452 & -779 & THMabc & -124 & -395 & -519 & -406 & -735 & -1141 \\
\hline D-LACt $2_{\leftarrow}$ & -452 & -327 & -779 & -327 & -452 & -779 & SBTpts & -2 & -14 & -16 & -20 & -44 & -64 \\
\hline GLYCLTt $2 r_{\rightarrow} \rightarrow$ & -454 & -328 & -782 & -328 & -454 & -782 & SERt4 & -15 & -17 & -32 & -17 & -15 & -32 \\
\hline GLYCLTt $2 r_{\leftarrow}$ & -454 & -328 & -782 & -328 & -454 & -782 & THRt4 & -13 & -14 & -27 & -14 & -13 & -27 \\
\hline L-LACt $2 r \rightarrow$ & -452 & -328 & -780 & -328 & -452 & -780 & TAURabc & -123 & -395 & -518 & -406 & -734 & -1140 \\
\hline $\mathrm{L}-\mathrm{LACt} 2 r_{\leftarrow}$ & -452 & -328 & -780 & -328 & -452 & -780 & THRt $2 r_{\rightarrow}$ & -454 & -330 & -784 & -330 & -454 & -784 \\
\hline LYSt $2 r \rightarrow$ & -453 & -328 & -781 & -328 & -453 & -781 & THRt $2 r_{\leftarrow}$ & -454 & -330 & -784 & -330 & -454 & -784 \\
\hline LYSt $2 r_{\leftarrow}$ & -453 & -328 & -781 & -328 & -453 & -781 & TRPt2r $\rightarrow$ & -453 & -326 & -779 & -326 & -453 & -779 \\
\hline MALTPTabc & -127 & -398 & -525 & -409 & -738 & -1147 & TRPt $2 r_{\leftarrow}$ & -453 & -326 & -779 & -326 & -453 & -779 \\
\hline MALTabc & -124 & -399 & -523 & -410 & -735 & -1145 & $\mathrm{Kt} 2 \mathrm{r} \rightarrow$ & -451 & -326 & -777 & -326 & -451 & -777 \\
\hline MALTTTRabc & -126 & -397 & -523 & -408 & -737 & -1145 & $\mathrm{Kt} 2 \mathrm{r}_{\leftarrow}$ & -451 & -326 & -777 & -326 & -451 & -777 \\
\hline MALTHXabc & -127 & -398 & -525 & -409 & -738 & -1147 & TYRt2r $\rightarrow$ & -451 & -327 & -778 & -327 & -451 & -778 \\
\hline MALTTRabc & -124 & -396 & -520 & -407 & -735 & -1142 & TYRt $2 r_{\leftarrow}$ & -451 & -327 & -778 & -327 & -451 & -778 \\
\hline FRUpts2 & -3 & -22 & -25 & -22 & -52 & -74 & GLYC3Pabc & -132 & -401 & -533 & -412 & -743 & -1155 \\
\hline MANpts & -2 & -16 & -18 & -20 & -46 & -66 & MAN6Pt6_2 & -149 & -38 & -187 & -38 & -149 & -187 \\
\hline GAMpts & -2 & -15 & -17 & -20 & -46 & -66 & G6Pt6_2 & -154 & -40 & -194 & -40 & -154 & -194 \\
\hline MELIBt 2 & -449 & -325 & -774 & -325 & -449 & -774 & FUCPt6_2 & -146 & -36 & -182 & -36 & -146 & -182 \\
\hline METabc & -125 & -395 & -520 & -406 & -736 & -1142 & URAt2 & -454 & -329 & -783 & -329 & -454 & -783 \\
\hline METDabc & -123 & -394 & -517 & -405 & -734 & -1139 & XTSNt2r $\rightarrow$ & -452 & -326 & -778 & -326 & -452 & -778 \\
\hline GALabc & -127 & -396 & -523 & -407 & -738 & -1145 & XTSNt2 $r_{\leftarrow}$ & -452 & -326 & -778 & -326 & -452 & -778 \\
\hline INDOLEt $2 r \rightarrow$ & -452 & -327 & -779 & -327 & -452 & -779 & INSt2r $\rightarrow$ & -454 & -328 & -782 & -328 & -454 & -782 \\
\hline INDOLEt $2 r_{\leftarrow}$ & -452 & -327 & -779 & -327 & -452 & -779 & INSt $2 r_{\leftarrow}$ & -454 & -328 & -782 & -328 & -454 & -782 \\
\hline ACNAMt2 & -449 & -325 & -774 & -325 & -449 & -774 & ADNt2r $\rightarrow$ & -454 & -330 & -784 & -330 & -454 & -784 \\
\hline N03t7 & -4 & -5 & -9 & -5 & -4 & -9 & ADNt $2 r_{\leftarrow}$ & -454 & -330 & -784 & -330 & -454 & -784 \\
\hline $\mathrm{NO} 2 \mathrm{t} 2 \mathrm{r} \rightarrow$ & -453 & -327 & -780 & -327 & -453 & -780 & CYTDt $2 r \rightarrow$ & -452 & -328 & -780 & -328 & -452 & -780 \\
\hline N02t $2 r_{\leftarrow}$ & -453 & -327 & -780 & -327 & -453 & -780 & CYTDt2r $r_{\leftarrow}$ & -452 & -328 & -780 & -328 & -452 & -780 \\
\hline NAt3_2 & -457 & -332 & -789 & -332 & -457 & -789 & THMDt $2 r \rightarrow$ & -453 & -328 & -781 & -328 & -453 & -781 \\
\hline NAt3_1.5 & -457 & -332 & -789 & -332 & -457 & -789 & THMDt $2 r_{\leftarrow}$ & -453 & -328 & -781 & -328 & -453 & -781 \\
\hline GSNt2 & -452 & -326 & -778 & -326 & -452 & -778 & URIt2r $\rightarrow$ & -454 & -328 & -782 & -328 & -454 & -782 \\
\hline DGSNt2 & -452 & -325 & -777 & -325 & -452 & -777 & URIt $2 r_{\leftarrow}$ & -454 & -328 & -782 & -328 & -454 & -782 \\
\hline INSt2 & -454 & -328 & -782 & -328 & -454 & -782 & XYLt2 & -451 & -326 & -777 & -326 & -451 & -777 \\
\hline DINSt2 & -451 & -325 & -776 & -325 & -451 & -776 & XYLabc & -125 & -396 & -521 & -407 & -736 & -1143 \\
\hline ADNt2 & -454 & -330 & -784 & -330 & -454 & -784 & CHLt $2 r_{\rightarrow}$ & -452 & -326 & -778 & -326 & -452 & -778 \\
\hline URIt2 & -454 & -328 & -782 & -328 & -454 & -782 & CHLt $2 r_{\leftarrow}$ & -452 & -326 & -778 & -326 & -452 & -778 \\
\hline CYTDt2 & -452 & -328 & -780 & -328 & -452 & -780 & $\operatorname{ADEt} 2 r_{\rightarrow}$ & -455 & -329 & -784 & -329 & -455 & -784 \\
\hline DCYTt2 & -450 & -325 & -775 & -325 & -450 & -775 & $\mathrm{ADEt} 2 r_{\leftarrow}$ & -455 & -329 & -784 & -329 & -455 & -784 \\
\hline DURIt2 & -452 & -326 & -778 & -326 & -452 & -778 & RIBabc & -123 & -395 & -518 & -406 & -734 & -1140 \\
\hline DADNt2 & -451 & -326 & -777 & -326 & -451 & -777 & $\mathrm{PSCVT}_{\rightarrow}$ & -4 & -37 & -41 & -20 & -146 & -166 \\
\hline THMDt2 & -453 & -328 & -781 & -328 & -453 & -781 & $\mathrm{PSCVT}_{\leftarrow}$ & -146 & -20 & -166 & -37 & -4 & -41 \\
\hline PNTOt4 & -9 & -9 & -18 & -9 & -9 & -18 & DHQS & 0 & -36 & -36 & 0 & -147 & -147 \\
\hline PIt2r $\rightarrow$ & -595 & -360 & -955 & -360 & -595 & -955 & CHORS & 0 & -40 & -40 & -1 & -146 & -147 \\
\hline PIt2r $\leftarrow$ & -595 & -360 & -955 & -360 & -595 & -955 & $\mathrm{DHQD}_{\rightarrow}$ & -1 & -234 & -235 & 0 & -87 & -87 \\
\hline NMNP & -2 & -4 & -6 & -4 & -2 & -6 & $\mathrm{DHQD}_{\leftarrow}$ & -87 & 0 & -87 & -234 & -1 & -235 \\
\hline PTRCabc & -129 & -400 & -529 & -411 & -740 & -1151 & SHK3Dr $\rightarrow$ & -467 & -19 & -486 & -368 & -44 & -412 \\
\hline SPMDabc & -125 & -397 & -522 & -408 & -736 & -1144 & $\mathrm{SHK} \mathrm{D} r_{\leftarrow}$ & -44 & -368 & -412 & -19 & -467 & -486 \\
\hline PTRCORNt $7 \rightarrow$ & -12 & -13 & -25 & -13 & -12 & -25 & DDPA & -91 & -36 & -127 & -256 & -146 & -402 \\
\hline PTRCORNt $7 \leftarrow$ & -12 & -13 & -25 & -13 & -12 & -25 & SHKK & -37 & -358 & -395 & -173 & -589 & -762 \\
\hline PTRCt2r $\rightarrow$ & -455 & -330 & -785 & -330 & -455 & -785 & PPNDH & -449 & -241 & -690 & -325 & -145 & -470 \\
\hline PTRCt $2 r_{\leftarrow}$ & -455 & -330 & -785 & -330 & -455 & -785 & CHORM & 0 & -1 & -1 & -4 & 0 & -4 \\
\hline $\mathrm{PROt} 2 r_{\rightarrow}$ & -453 & -328 & -781 & -328 & -453 & -781 & TRPAS2 $\rightarrow$ & -90 & -24 & -114 & -235 & -80 & -315 \\
\hline $\mathrm{PROt} 2 \mathrm{r}_{\leftarrow}$ & -453 & -328 & -781 & -328 & -453 & -781 & TRPAS $2_{\leftarrow}$ & -80 & -235 & -315 & -24 & -90 & -114 \\
\hline PROabc & -127 & -398 & -525 & -409 & -738 & -1147 & TRPS2 & -10 & -235 & -245 & -12 & -90 & -102 \\
\hline PIabc & -269 & -394 & -663 & -441 & -734 & -1175 & TRPS3 & 0 & -12 & -12 & -1 & -15 & -16 \\
\hline ACMANApts & -3 & -14 & -17 & -21 & -44 & -65 & TRPS1 & -7 & -244 & -251 & -10 & -102 & -112 \\
\hline MNLpts & -2 & -14 & -16 & -20 & -44 & -64 & PRAIi & 0 & 0 & 0 & 0 & 0 & 0 \\
\hline SUCpts & -2 & -14 & -16 & -20 & -43 & -63 & IGPS & -449 & -242 & -691 & -324 & -144 & -468 \\
\hline GLCpts & -19 & -18 & -37 & -23 & -51 & -74 & ANS & -1 & -366 & -367 & -16 & -526 & -542 \\
\hline FRUpts & -3 & -14 & -17 & -22 & -43 & -65 & ANPRT & -1 & -7 & -8 & -11 & -72 & -83 \\
\hline TREpts & -3 & -15 & -18 & -22 & -44 & -66 & PPND & -41 & -46 & -87 & -51 & -106 & -157 \\
\hline PROt4 & -12 & -12 & -24 & -12 & -12 & -24 & TYRTA $\rightarrow$ & -19 & -28 & -47 & -23 & -35 & -58 \\
\hline
\end{tabular}




\begin{tabular}{|c|c|c|c|c|c|c|c|c|c|c|c|c|c|}
\hline TYRTA $_{\leftarrow}$ & -35 & -23 & -58 & -28 & -19 & -47 & KARA2i & -466 & -18 & -484 & -367 & -44 & -411 \\
\hline PHETA1 $\rightarrow$ & -19 & -28 & -47 & -22 & -35 & -57 & KARA1i & -466 & -18 & -484 & -367 & -44 & -411 \\
\hline PHETA $_{\leftarrow}$ & -35 & -22 & -57 & -28 & -19 & -47 & DHAD2 & 0 & -233 & -233 & 0 & -87 & -87 \\
\hline ATPM & -123 & -394 & -517 & -405 & -734 & -1139 & DHAD1 & 0 & -236 & -236 & 0 & -87 & -87 \\
\hline BETALDHx & -126 & -364 & -490 & -284 & -500 & -784 & ILETA $\rightarrow$ & -20 & -28 & -48 & -23 & -35 & -58 \\
\hline BETALDHy & -129 & -369 & -498 & -252 & -470 & -722 & ILETA $_{\leftarrow}$ & -35 & -23 & -58 & -28 & -20 & -48 \\
\hline $\mathrm{HCO} 3 \mathrm{E}_{\rightarrow}$ & -144 & -328 & -472 & -241 & -450 & -691 & $\mathrm{VALTA}_{\rightarrow}$ & -21 & -31 & -52 & -23 & -35 & -58 \\
\hline $\mathrm{HCO} \mathrm{E}_{\leftarrow}$ & -450 & -241 & -691 & -328 & -144 & -472 & VALTA $_{\leftarrow}$ & -35 & -23 & -58 & -31 & -21 & -52 \\
\hline CYANST & 1 & -323 & -322 & -1 & -452 & -453 & LEUTAi & -34 & -22 & -56 & -28 & -20 & -48 \\
\hline CAT & -4 & -253 & -257 & -1 & -90 & -91 & IPPS & -100 & -345 & -445 & -252 & -473 & -725 \\
\hline SELNPS & -122 & -41 & -163 & -405 & -179 & -584 & IPMD & -41 & -362 & -403 & -51 & -496 & -547 \\
\hline SPODM & -448 & -21 & -469 & -324 & -8 & -332 & OMCDC & -449 & -8 & -457 & -324 & -58 & -382 \\
\hline ACONMT & -1 & 0 & -1 & -7 & -6 & -13 & IPPMIa $_{\rightarrow}$ & 0 & -234 & -234 & -1 & -87 & -88 \\
\hline THRD_L & -5 & -8 & -13 & -6 & -34 & -40 & $\mathrm{IPPMIa}_{\leftarrow}$ & -87 & -1 & -88 & -234 & 0 & -234 \\
\hline ACHBS & -492 & -8 & -500 & -339 & -58 & -397 & IPPMIb $\rightarrow_{\rightarrow}$ & -87 & 0 & -87 & -234 & -1 & -235 \\
\hline ACLS & -492 & -8 & -500 & -338 & -58 & -396 & IPPMIb $_{\leftarrow}$ & -1 & -234 & -235 & 0 & -87 & -87 \\
\hline
\end{tabular}

